\title{
Spin-Orbital Order Modified by Orbital Dilution in Transition-Metal Oxides: From Spin Defects to Frustrated Spins Polarizing Host Orbitals
}

\author{
Wojciech Brzezicki, ${ }^{1,2}$ Andrzej M. Oleś, ${ }^{3,1}$ and Mario Cuoco ${ }^{2}$ \\ ${ }^{1}$ Marian Smoluchowski Institute of Physics, Jagiellonian University, \\ prof. S. Łojasiewicza 11, PL-30348 Kraków, Poland \\ ${ }^{2}$ CNR-SPIN, IT-84084 Fisciano (SA), Italy, and Dipartimento di Fisica “E. R. Caianiello," \\ Universitá di Salerno, IT-84084 Fisciano (SA), Italy \\ ${ }^{3}$ Max-Planck-Institut für Festkörperforschung, Heisenbergstrasse 1, D-70569 Stuttgart, Germany \\ (Received 7 August 2014; revised manuscript received 24 December 2014; published 27 March 2015)
}

We investigate the changes in spin and orbital patterns induced by magnetic transition-metal ions without an orbital degree of freedom doped in a strongly correlated insulator with spin-orbital order. In this context, we study the $3 d$ ion substitution in $4 d$ transition-metal oxides in the case of $3 d^{3}$ doping at either $3 d^{2}$ or $4 d^{4}$ sites, which realizes orbital dilution in a Mott insulator. Although we concentrate on this doping case as it is known experimentally and more challenging than other oxides due to finite spin-orbit coupling, the conclusions are more general. We derive the effective $3 d-4 d$ (or $3 d-3 d$ ) superexchange in a Mott insulator with different ionic valencies, underlining the emerging structure of the spin-orbital coupling between the impurity and the host sites, and demonstrate that it is qualitatively different from that encountered in the host itself. This derivation shows that the interaction between the host and the impurity depends in a crucial way on the type of doubly occupied $t_{2 g}$ orbital. One finds that in some cases, due to the quench of the orbital degree of freedom at the $3 d$ impurity, the spin and orbital order within the host is drastically modified by doping. The impurity either acts as a spin defect accompanied by an orbital vacancy in the spin-orbital structure when the host-impurity coupling is weak or favors doubly occupied active orbitals (orbital polarons) along the $3 d-4 d$ bond leading to antiferromagnetic or ferromagnetic spin coupling. This competition between different magnetic couplings leads to quite different ground states. In particular, for the case of a finite and periodic $3 d$ atom substitution, it leads to striped patterns either with alternating ferromagnetic or antiferromagnetic domains or with islands of saturated ferromagnetic order. We find that magnetic frustration and spin degeneracy can be lifted by the quantum orbital flips of the host, but they are robust in special regions of the incommensurate phase diagram. Orbital quantum fluctuations modify quantitatively spin-orbital order imposed by superexchange. In contrast, the spin-orbit coupling can lead to anisotropic spin and orbital patterns along the symmetry directions and cause a radical modification of the order imposed by the spin-orbital superexchange. Our findings are expected to be of importance for future theoretical understanding of experimental results for $4 d$ transition-metal oxides doped with $3 d^{3}$ ions. We suggest how the local or global changes of the spin-orbital order induced by such impurities could be detected experimentally.

DOI: 10.1103/PhysRevX.5.011037

\section{INTRODUCTION}

The studies of strongly correlated electrons in transitionmetal oxides (TMOs) focus traditionally on $3 d$ materials [1], mainly because of high-temperature superconductivity discovered in cuprates and more recently in iron pnictides, and also because of colossal magnetoresistance manganites. The competition of different and complex types of

Published by the American Physical Society under the terms of the Creative Commons Attribution 3.0 License. Further distribution of this work must maintain attribution to the author(s) and the published article's title, journal citation, and DOI.
Subject Areas: Magnetism, Materials Science, Strongly Correlated Materials order is ubiquitous in strongly correlated TMOs mainly due to coupled spin-charge orbitals where frustrated exchange competes with the kinetic energy of charge carriers. The best-known example is spin-charge competition in cuprates, where spin, charge, and superconducting orders intertwine [2] and stripe order emerges in the normal phase as a compromise between the magnetic and kinetic energy $[3,4]$. Remarkable evolution of the stripe order under increasing doping is observed [5] and could be reproduced by the theory based on the extended Hubbard model [6]. Hole doping in cuprates corresponds to the removal of the spin degree of freedom. Similarly, hole doping in the simplest system with the orbital order in the $d^{1}$ configuration removes 
locally orbital degrees of freedom and generates stripe phases that involve orbital polarons [7]. It was predicted recently that orbital domain walls in bilayer manganites should be partially charged as a result of competition between orbital-induced strain and Coulomb repulsion [8], which opens a new route toward charge-orbital physics in TMOs. We will show below that the stripelike order may also occur in doped spin-orbital systems. These systems are very challenging, and their doping leads to very complex and yet unexplored spin-orbital-charge phenomena [9].

A prerequisite to the phenomena with spin-orbitalcharge-coupled degrees of freedom is the understanding of undoped systems [10], where the low-energy physics and spin-orbital order are dictated by effective spin-orbital superexchange [11-13] and compete with spin-orbital quantum fluctuations [14-16]. Although ordered states occur in many cases, the most intriguing are quantum phases such as spin [17] or orbital [18] liquids. Recent experiments on a copper oxide $\mathrm{Ba}_{3} \mathrm{CuSb}_{2} \mathrm{O}_{9}[19,20]$ have triggered renewed efforts in a fundamental search for a quantum spin-orbital liquid [21-24], where spin-orbital order is absent and electron spins are randomly choosing orbitals which they occupy. A signature of strong quantum effects in a spin-orbital system is a disordered state that persists down to very low temperatures. A good example of such a disordered spin-orbital liquid state is also $\mathrm{FeSc}_{2} \mathrm{~S}_{4}$, which does not order in spite of finite Curie-Weiss temperature $\Theta_{\mathrm{CW}}=-45 \mathrm{~K}$ [25] but shows instead signatures of quantum criticality $[26,27]$.

Spin-orbital interactions may be even more challengingforinstance, previous attempts to find a spin-orbital liquid in the Kugel-Khomskii model [14] or in $\mathrm{LiNiO}_{2}$ [28] turned out to be unsuccessful. In fact, in the former case, certain types of exotic spin order arise as a consequence of frustrated and entangled spin-orbital interactions [29,30], and a spin-orbital entangled resonating valence-bond state was recently shown to be a quantum superposition of striped spin-singlet covering on a square lattice [31]. In contrast, spin and orbital superexchange have different energy scales and orbital interactions in $\mathrm{LiNiO}_{2}$ are much stronger and dominated by frustration [32]. Hence, the reasons behind the absence of magnetic long-range order are more subtle [33]. In all these cases, orbital fluctuations play a prominent role and spin-orbital entanglement [34] determines the ground state.

The role of charge carriers in spin-orbital systems is under very active investigation at present. In doped $\mathrm{La}_{1-x}(\mathrm{Sr}, \mathrm{Ca})_{x} \mathrm{MnO}_{3}$ manganites, several different types of magnetic order compete with one another and occur at increasing hole doping [35-37]. Undoped $\mathrm{LaMnO}_{3}$ is an antiferromagnetic (AF) Mott insulator, with large $S=2$ spins for $3 d^{4}$ ionic configurations of $\mathrm{Mn}^{3+}$ ions stabilized by Hund's exchange, coupled via the spin-orbital superexchange due to $e_{g}$ and $t_{2 g}$ electron excitations [38]. The orbital $e_{g}$ degree of freedom is removed by hole doping when $\mathrm{Mn}^{3+}$ ions are generated, and this removal of the orbital degree of freedom requires careful modeling in the theory that takes into account both $3 d^{4}$ and $3 d^{3}$ electronic configurations of $\mathrm{Mn}^{3+}$ and $\mathrm{Mn}^{4+}$ ions [39-44]. In fact, the orbital order changes radically with increasing doping in $\mathrm{La}_{1-x}(\mathrm{Sr}, \mathrm{Ca})_{x} \mathrm{MnO}_{3}$ systems at the magnetic phase transitions between different types of magnetic order [37], as well as at $\mathrm{La}_{0.7} \mathrm{Ca}_{0.3} \mathrm{MnO}_{3} / \mathrm{BiFeO}_{3}$ heterostructures, where it offers a new route to enhancing multiferroic functionality [45]. The double-exchange mechanism [46] triggers the ferromagnetic (FM) metallic phase at sufficient doping; in this phase, the spin and orbital degrees of freedom decouple and spin excitations are explained by the orbital liquid [47,48]. Because of distinct magnetic and kinetic energy scales, even low doping may suffice for a drastic change in the magnetic order, as observed in electron-doped manganites [49].

A rather unique example of a spin-orbital system with strongly fluctuating orbitals, as predicted in the theory [50-52] and seen experimentally [53-55], are the perovskite vanadates with competing spin-orbital order [56]. In these $t_{2 g}$ systems, $x y$ orbitals are filled by one electron and the orbital order of active $\{y z, z x\}$ orbitals is strongly influenced by doping with $\mathrm{Ca}(\mathrm{Sr})$ ions that replace the $\mathrm{Y}(\mathrm{La})$ ones in $\mathrm{YVO}_{3}\left(\mathrm{LaVO}_{3}\right)$. In this case, finite spin-orbit coupling modifies the spin-orbital phase diagram [57]. In addition, the $\mathrm{AF}$ order switches easily from the $G$-type $\mathrm{AF}(G-\mathrm{AF})$ to $C$-type $\mathrm{AF}(C$-AF) order in the presence of charge defects in $\mathrm{Y}_{1-x} \mathrm{Ca}_{x} \mathrm{VO}_{3}$. Already at low $x \simeq 0.02$ doping, the spinorbital order changes and spectral weight is generated within the Mott-Hubbard gap [58]. Although one might imagine that the orbital degree of freedom is thereby removed, a closer inspection shows that this is not the case as the orbitals are polarized by charge defects [59] and readjust near them [60]. Removing the orbital degree of freedom in vanadates would only be possible by electron doping, generating instead $d^{3}$ ionic configurations, but such a doping by charge defects would be very different from the doping by transition-metal ions of the same valence considered below.

Also, in $4 d$ materials, spin-orbital physics plays a role [61], as, for instance, in $\mathrm{Ca}_{2-x} \mathrm{Sr}_{x} \mathrm{RuO}_{4}$ systems with $\mathrm{Ru}^{4+}$ ions in a $4 d^{4}$ configuration [62-66]. Recently, it has been shown that unconventional magnetism is possible for $\mathrm{Ru}^{4+}$ and similar ions where spin-orbit coupling plays a role $[67,68]$. Surprisingly, these systems are not similar to manganites but to vanadates where one also finds ions with active $t_{2 g}$ orbitals. In the case of ruthenates, the $t_{2 g}^{4}$ $\mathrm{Ru}^{4+}$ ions have low $S=1$ spin as the splitting between the $t_{2 g}$ and $e_{g}$ levels is large. Thus, the undoped $\mathrm{Ca}_{2} \mathrm{RuO}_{4}$ is a hole analogue of a vanadate [50,51], with $t_{2 g}$ orbital degrees of freedom and $S=1$ spin per site in both cases. Such systems give new opportunities to investigate spin-orbital entangled states in the $t_{2 g}$ system, observed recently by angle-resolved photoemission [69]. 
Here, we focus on a novel and very different doping from all those considered above, namely, on a substitutional doping by other magnetic ions in a plane built by transitionmetal and oxygen ions, for instance, in the $(a, b)$ plane of a monolayer or in perovskite ruthenates or vanadates. In this study, we are interested primarily in doping of a TMO with $t_{2 g}$ orbital degrees of freedom, where doped magnetic ions have no orbital degree of freedom and realize orbital dilution. In addition, we deal with the simpler case of $3 d$ doped ions where we can neglect spin-orbit interaction, which should not be ignored for $4 d$ ions. We emphasize that in contrast to manganites where holes within $e_{g}$ orbitals participate in transport and are responsible for the colossal magnetoresistance, such doped holes are immobile due to the ionic potential at $3 d$ sites and form defects in the spin-orbital order of a Mott insulator. We encounter here a different situation from the dilution effects in the 2D $e_{g}$ orbital system considered so far [70] as we deal with magnetic ions at doped sites. It is challenging to investigate how such impurities modify locally or globally the spin-orbital order of the host.

The doping that realizes this paradigm is by either $\mathrm{Mn}^{4+}$ or $\mathrm{Cr}^{3+}$ ions with large $S=3 / 2$ spins stabilized by Hund's exchange, and orbital dilution occurs either in a TMO with $d^{2}$ ionic configuration as in the vanadium perovskites or in $4 d$ Mott insulators as in ruthenates. It has been shown that dilute $\mathrm{Cr}$ doping for $\mathrm{Ru}$ reduces the temperature of the orthorhombic distortion and induces FM behavior in $\mathrm{Ca}_{2} \mathrm{Ru}_{1-x} \mathrm{Cr}_{x} \mathrm{O}_{4}$ (with $0<x<0.13$ ) [71]. It also induces surprisingly negative volume thermal expansion via spinorbital order. Such defects, on one hand, can weaken the spin-orbital coupling in the host but, on the other hand, may open a new channel of interaction between the spin and orbital degrees of freedom through the host-impurity exchange; see Fig. 1. The consequences of such doping are yet unexplored and are expected to open a new route in the research on strongly correlated oxides.

The physical examples for the present theory are the insulating phases of $3 d-4 d$ hybrid structures, where doping happens at $d^{4}$ transition-metal sites, and the value of the spin is locally changed from $S=1$ to $S=3 / 2$. As a demonstration of the highly nontrivial physics emerging in $3 d-4 d$ oxides, remarkable effects have already been observed, for instance, when $\mathrm{Ru}$ ions are replaced by $\mathrm{Mn}, \mathrm{Ti}, \mathrm{Cr}$, or other $3 d$ elements. The role of Mn doping in the $\mathrm{SrRuO}$ Ruddlesden-Popper series is strongly linked to the dimensionality through the number $n$ of $\mathrm{RuO}_{2}$ layers in the unit cell. The $\mathrm{Mn}$ doping of the $\mathrm{SrRuO}_{3}$ cubic member drives the system from the itinerant FM state to an insulating AF configuration in a continuous way via a possible unconventional quantum phase transition [72]. Doping by $\mathrm{Mn}$ ions in $\mathrm{Sr}_{3} \mathrm{Ru}_{2} \mathrm{O}_{7}$ leads to a metal-toinsulator transition and $\mathrm{AF}$ long-range order for more than 5\% Mn concentration [73]. Subtle orbital rearrangement can occur at the Mn site, as, for instance, the inversion of

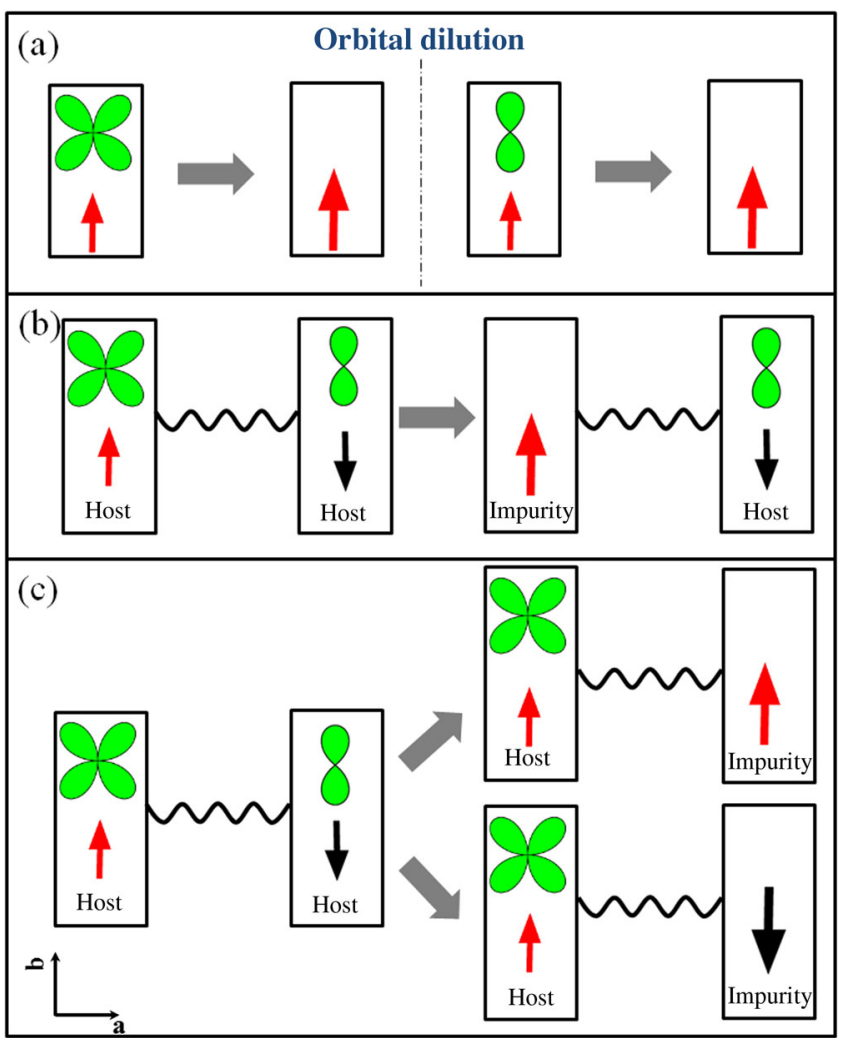

FIG. 1. (a) Schematic view of the orbital dilution when the $3 d^{3}$ ion with no orbital degree of freedom and spin $S=3 / 2$ substitutes the $4 d^{4}$ one with spin $S=1$ on a bond having specific spin and orbital character in the host (gray arrows). Spins are shown by red arrows, and doubly occupied $t_{2 g}$ orbitals (doublons) are shown by green symbols for the $a$ and $c$ orbitals, respectively. (b) If an inactive orbital along the bond is removed by doping, the total spin exchange is AF. (c) On the contrary, active orbitals at the host site can lead to either FM (top) or AF (bottom) exchange coupling, depending on the energy-level mismatch and difference in the Coulomb couplings between the impurity and the host. We show the case when the host site is unchanged in the doping process.

the crystal field in the $e_{g}$ sector observed via x-ray absorption spectroscopy [74]. Neutron-scattering studies indicate the occurrence of an unusual $E$-type antiferromagnetism in doped systems (planar order with FM zigzag chains with AF order between them) with moments aligned along the $c$ axis within a single bilayer [75].

Furthermore, the more extended $4 d$ orbitals would a priori suggest a weaker correlation than in $3 d$ TMOs due to a reduced ratio between the intra-atomic Coulomb interaction and the electron bandwidth. Nevertheless, the (effective) $d$ bandwidth is reduced by the changes in the $3 d-2 p-3 d$ bond angles in distorted structures that typically arise in these materials, bringing them on the verge of a metal-insulator transition [76] or even into the Mott-insulating state with spin-orbital order; see Fig. 2. Hence, not only do $4 d$ materials share common features with $3 d$ systems, but 


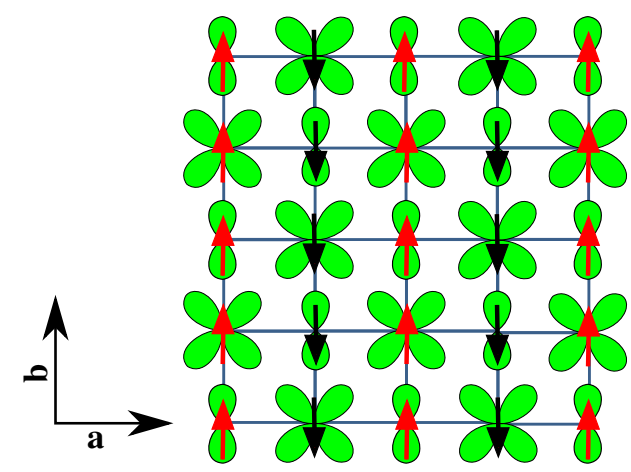

FIG. 2. Schematic view of $C$-AF spin order coexisting with $G$-AO orbital order in the $(a, b)$ plane of an undoped Mott insulator with $4 d^{4}$ ionic configurations. Spins are shown by arrows, while doubly occupied $x y$ and $y z$ orbitals ( $c$ and $a$ doublons; see the text) form a checkerboard pattern. Equivalent spin-orbital order is realized for $\mathrm{V}^{3+}$ ions in the $(b, c)$ planes of $\mathrm{LaVO}_{3}$ [56], with orbitals standing for empty orbitals (holes).

they are also richer due to their sensitivity to the lattice structure and to relativistic effects due to larger spin-orbit [77] or other magnetocrystalline couplings.

To simplify the analysis, we assume that on-site Coulomb interactions are so strong that charge degrees of freedom are projected out, and only virtual charge transfer can occur between $3 d$ and $4 d$ ions via the oxygen ligands. For convenience, we define the orbital degree of freedom as a doublon (double occupancy) in the $t_{2 g}^{4}$ configuration. The above $3 d$ doping then leads effectively to the removal of a doublon in one of the $t_{2 g}$ orbitals which we label as $\{a, b, c\}$ (this notation is introduced in Ref. [16] and explained below) and to replacing it by a $t_{2 g}^{3}$ ion. To our knowledge, this is the only example of removing the orbital degree of freedom in the $t_{2 g}$ manifold realized so far, and below, we investigate possible consequences of this phenomenon. Another possibility of orbital dilution that awaits experimental realization would occur when a $t_{2 g}$ degree of freedom is removed by replacing a $d^{2}$ ion by a $d^{3}$ one, as, for instance, by $\mathrm{Cr}^{3+}$ doping in a vanadate-here, a doublon is an empty $t_{2 g}$ orbital, i.e., filled by two holes.

Before presenting the details of the quantitative analysis, let us concentrate on the main idea of the superexchange modified by doping in a spin-orbital system. The $d^{3}$ ions have singly occupied all three $t_{2 g}$ orbitals and $S=3 / 2$ spins due to Hund's exchange. While a pair of $d^{3}$ ions, e.g., in $\mathrm{SrMnO}_{3}$, is coupled by AF superexchange [48], the superexchange for the $d^{3}-d^{4}$ bond has a rather rich structure and may also be FM. The spin exchange depends, then, on whether the orbital degree of freedom is active and participates in charge excitations along a considered bond, or electrons of the doublon cannot move along this bond due to the symmetry of the $t_{2 g}$ orbital, as explained in Fig. 1. This qualitative difference to systems without active orbital degrees of freedom is investigated in detail in Sec. II.
The main outcomes of our analysis are (i) the determination of the effective spin-orbital exchange Hamiltonian describing the low-energy sector for the $3 d-4 d$ hybrid structure, (ii) establishing that a $3 d^{3}$ impurity without an orbital degree of freedom modifies the orbital order in the $4 d^{4}$ host, (iii) providing the detailed way how the microscopic spin-orbital order within the $4 d^{4}$ host is modified around the $3 d^{3}$ impurity, and (iv) suggesting possible spinorbital patterns that arise due to periodic and finite substitution (doping) of $4 d$ atoms in the host by $3 d$ ones. The emerging physical scenario is that the $3 d$ impurity acts as an orbital vacancy when the host-impurity coupling is weak and as an orbital polarizer of the bond's active $t_{2 g}$ doublon configurations when it is strongly coupled to the host. The tendency to polarize the host orbitals around the impurity turns out to be robust and independent of spin configuration. Otherwise, it is the resulting orbital arrangement around the impurity and the strength of Hund's coupling at the impurity that set the character of the host-impurity magnetic exchange.

The remainder of the paper is organized as follows. In Sec. II, we introduce the effective model describing the spin-orbital superexchange at the $3 d-4 d$ bonds, which serves to investigate the changes of spin and orbital order around individual impurities and at finite doping. We arrive at a rather general formulation that emphasizes the impurity orbital degree of freedom, being a doublon, and present some technical details of the derivation in Appendix A. The strategy we adopt is to analyze first the ground-state properties of a single $3 d^{3}$ impurity surrounded by $4 d^{4}$ atoms by investigating how the spin-orbital pattern in the host may be modified at the nearest-neighbor (NN) sites to the $3 d$ atom. This study is performed for different spinorbital patterns of the $4 d$ host with special emphasis on the alternating $\mathrm{FM}$ chains $(C$-AF order) which coexist with $G$-type alternating orbital ( $G$-AO) order; see Fig. 2 . We address the impurity problem within the classical approximation in Sec. III A. As explained in Sec. III B, there are two nonequivalent cases that depend on the precise modification of the orbital order by the $3 d$ impurity, doped either to replace a doublon in the $a$ orbital (Sec. III C) or the one in the $c$ orbital (Sec. III D).

Starting from the single-impurity solution, we next address periodic arrangements of $3 d$ atoms at different concentrations. We demonstrate that the spin-orbital order in the host can be radically changed by the presence of impurities, leading to striped patterns with alternating FM or AF domains and islands of fully FM states. In Sec. IV A, we consider the modifications of spin-orbital order that arise at periodic doping with macroscopic concentration. Here, we limit ourselves to two representative cases: (i) commensurate $x=1 / 8$ doping in Sec. IV B and (ii) two doping levels $x=1 / 5$ and $x=1 / 9$ being incommensurate with underlying two-sublattice order (Fig. 2), which implies simultaneous doping at two sublattices, i.e., at 
both the $a$ - and $c$-doublon sites, as presented in Secs. IV C and IV D. Finally, in Sec. VA, we investigate the modifications of the classical phase diagram induced by quantum fluctuations, and in Secs. VB and VC, we discuss representative results obtained for finite spin-orbit coupling. (Calculation details of the treatment of spin-orbit interaction are presented in Appendix B.) The paper is concluded by a general discussion of possible emerging scenarios for the $3 d^{3}$ impurities in a $4 d^{4}$ host, a summary of the main results, and the perspective of future experimental investigations of orbital dilution in Sec. VI.

\section{THE SPIN-ORBITAL MODEL}

In this section, we consider a $3 d$ impurity in a strongly correlated $4 d$ TMO and derive the effective $3 d^{3}-4 d^{4}$ spin-orbital superexchange. It follows from the coupling between $3 d$ and $4 d$ orbitals via oxygen $2 p$ orbitals due to the $p-d$ hybridization. In a strongly correlated system, it suffices to concentrate on a pair of atoms forming a bond $\langle i j\rangle$, as the effective interactions are generated by charge excitations $d_{i}^{4} d_{j}^{4} \leftrightharpoons d_{i}^{5} d_{j}^{3}$ along a single bond [12]. In the reference $4 d$ host, both atoms on the bond $\langle i j\rangle$ are equivalent and one considers

$$
H(i, j)=H_{t}(i, j)+H_{\text {int }}(i)+H_{\text {int }}(j) .
$$

The Coulomb interaction $H_{\text {int }}(i)$ is local at site $i$, and we describe it by the degenerate Hubbard model [78]; see below.

We implement a strict rule that the hopping within the $t_{2 g}$ sector is allowed in a TMO only between two neighboring orbitals of the same symmetry that are active along the bond direction $[15,79,80]$ and neglect the interorbital processes originating from the octahedral distortions such as rotation or tilting. Indeed, in ideal undistorted (perovskite or square lattice) geometry, the orbital flavor is conserved as long as the spin-orbit coupling may be neglected. The interorbital hopping elements are smaller by at least 1 order of magnitude and may be treated as corrections in cases where distortions play a role to the overall scenario established below.

The kinetic energy for a representative $3 d-2 p-4 d$ bond, i.e., after projecting out the oxygen degrees of freedom, is given by the hopping in the host $\propto t_{h}$ between sites $i$ and $j$

$$
H_{t}(i, j)=-t_{h} \sum_{\mu(\gamma), \sigma}\left(d_{i \mu \sigma}^{\dagger} d_{j \mu \sigma}+d_{j \mu \sigma}^{\dagger} d_{i \mu \sigma}\right) .
$$

Here, $d_{i \mu \sigma}^{\dagger}$ are the electron-creation operators at site $i$ in the spin-orbital state $(\mu \sigma)$. The bond $\langle i j\rangle$ points along one of the two crystallographic directions $\gamma=a, b$ in the twodimensional (2D) square lattice. Without distortions, only two out of three $t_{2 g}$ orbitals are active along each bond $\langle i j\rangle$ and contribute to $H_{t}(i, j)$, while the third orbital lies in the plane perpendicular to the $\gamma$ axis and thus the hopping via oxygen is forbidden by symmetry. The symmetry of the $t_{2 g}$ connectivity motivates a convenient notation as follows [15]:

$$
|a\rangle \equiv|y z\rangle, \quad|b\rangle \equiv|x z\rangle, \quad|c\rangle \equiv|x y\rangle,
$$

with the $t_{2 g}$ orbital inactive along a given direction $\gamma \in\{a, b, c\}$ labeled by the index $\gamma$. We consider a 2D square lattice with transition-metal ions connected via oxygen orbitals as in a $\mathrm{RuO}_{2}(a, b)$ plane of $\mathrm{Ca}_{2} \mathrm{RuO}_{4}$ $\left(\mathrm{SrRuO}_{3}\right)$. In this case, $|a\rangle(|b\rangle)$ orbitals are active along the $b(a)$ axis, while $|c\rangle$ orbitals are active along both the $a$ and $b$ axes.

To derive the superexchange in a Mott insulator, it is sufficient to consider a bond that connects nearest-neighbor sites $\langle i j\rangle \equiv\langle 12\rangle$. Below, we consider a bond between an impurity site $i=1$ occupied by a $3 d$ ion and a neighboring host $4 d$ ion at site $j=2$. The Hamiltonian for this bond can be then expressed in the following form:

$$
H(1,2)=H_{t}(1,2)+H_{\text {int }}(1)+H_{\text {int }}(2)+H_{\text {ion }}(2) .
$$

The total Hamiltonian contains the kinetic energy term $H_{t}(1,2)$ describing the electron-charge transfer via oxygen orbitals, the on-site interaction terms $H_{\text {int }}(m)$ for the $3 d(4 d)$ ion at site $m=1,2$, and the local potential of the $4 d$ atom $H_{\text {ion }}(2)$, which takes into account the mismatch of the energy-level structure between the two ( $4 d$ and $3 d$ ) atomic species and prevents valence fluctuations when the host is doped, even in the absence of local Coulomb interaction.

The kinetic energy in Eq. (4) is given by

$$
H_{t}(1,2)=-t \sum_{\mu(\gamma), \sigma}\left(d_{1 \mu \sigma}^{\dagger} d_{2 \mu \sigma}+d_{2 \mu \sigma}^{\dagger} d_{1 \mu \sigma}\right),
$$

where $d_{m \mu \sigma}^{\dagger}$ is the electron-creation operator at site $m=1,2$ in the spin-orbital state $(\mu \sigma)$. The bond $\langle 12\rangle$ points along one of the two crystallographic directions $\gamma=a, b$, and again the orbital flavor is conserved $[15,79,80]$.

The Coulomb interaction on an atom at site $m=1,2$ depends on two parameters [78]: (i) intraorbital Coulomb repulsion $U_{m}$ and (ii) Hund's exchange $J_{m}^{H}$. The label $m$ stands for the ion and distinguishes between these terms at the $3 d$ and $4 d$ ions, respectively. The interaction is expressed in the form

$$
\begin{aligned}
H_{\mathrm{int}}(m)= & U_{m} \sum_{\mu} n_{m \mu \uparrow} n_{m \mu \downarrow}-2 J_{m}^{H} \sum_{\mu<\nu} \vec{S}_{m \mu} \cdot \vec{S}_{m \nu} \\
& +\left(U_{m}-\frac{5}{2} J_{m}^{H}\right) \sum_{\substack{\mu<\nu \\
\sigma \sigma^{\prime}}} n_{m \mu \sigma} n_{m \nu \sigma^{\prime}} \\
& +J_{m}^{H} \sum_{\mu \neq \nu} d_{m \mu \uparrow}^{\dagger} d_{m \mu \downarrow}^{\dagger} d_{m \nu \downarrow} d_{m \nu \uparrow} \cdot
\end{aligned}
$$


The terms standing in the first line of Eq. (6) contribute to the magnetic instabilities in the degenerate Hubbard model [78] and decide about spin order, both in an itinerant system and in a Mott insulator. The remaining terms contribute to the multiplet structure and are of importance for the correct derivation of the superexchange that follows from charge excitations; see below.

Finally, we include a local potential on the $4 d$ atom that encodes the energy mismatch between the host and the impurity orbitals close to the Fermi level and prevents valence fluctuations on the $4 d$ ion due to the $3 d$ doping. This term has the following general structure:

$$
H_{\mathrm{ion}}(2)=I_{2}^{e}\left(4-\sum_{\mu, \sigma} n_{2 \mu \sigma}\right)^{2}
$$

with $\mu=a, b, c$.

The effective Hamiltonian for the low-energy processes is derived from $H(1,2)$ (4) by a second-order expansion for charge excitations generated by $H_{t}(1,2)$ and treating the remaining part of $H(1,2)$ as an unperturbed Hamiltonian. We are basically interested in virtual charge excitations in the manifold of degenerate ground states of a pair of $3 d$ and $4 d$ atoms on a bond; see Fig. 3. These quantum states are labeled as $\left\{e_{1}^{k}\right\}$ with $k=1, \ldots, 4$ and $\left\{e_{2}^{p}\right\}$ with $p=1, \ldots, 9$, and their number follows from the solution of the on-site quantum problem for the Hamiltonian $H_{\text {int }}(i)$. For the $3 d$ atom, the relevant states can be classified according to the four components of the total spin $S_{1}=3 / 2$ for the $3 d$ impurity atom at site $m=1$ and the three components of $S_{2}=1$ spin for the $4 d$ host atom at site $m=2$, and for the three different positions of the double occupied orbital (doublon). Thus, the effective Hamiltonian will contain spin products $\left(\vec{S}_{1} \cdot \vec{S}_{2}\right)$ between spin operators defined as

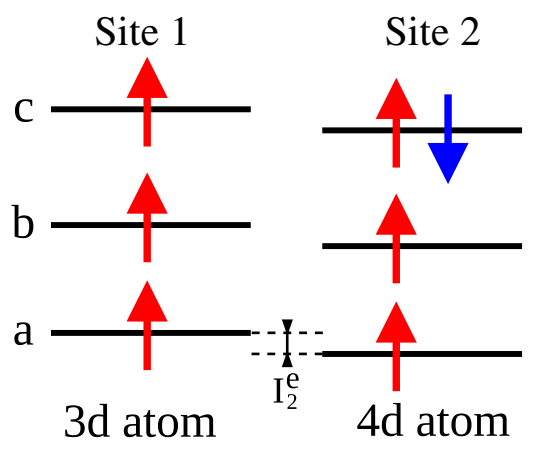

FIG. 3. Schematic representation of one configuration belonging to the manifold of 36 degenerate ground states for a representative $3 d-4 d$ bond as given by the local Coulomb Hamiltonian $H_{\text {int }}(m)(6)$ with $m=1,2$. The dominant exchange processes considered here are those that move one of the four electrons on the $4 d$ atom to the $3 d$ neighbor and back. The stability of the $3 d^{3}-4 d^{4}$ charge configurations is provided by the local potential energy $I_{2}^{e}$; see Eq. (7).

$$
\vec{S}_{m}=\frac{1}{2} \sum_{\gamma} d_{m \gamma \alpha}^{\dagger} \vec{\sigma}_{\alpha \beta} d_{m \gamma \beta}
$$

for $m=1,2$ sites and the operator of the doublon position at site $m=2$

$$
D_{2}^{(\gamma)}=\left(d_{2 \gamma \uparrow}^{\dagger} d_{2 \gamma \uparrow}\right)\left(d_{2 \gamma \downarrow}^{\dagger} d_{2 \gamma \downarrow}\right) .
$$

The doublon operator identifies the orbital $\gamma$ within the $t_{2 g}$ manifold of the $4 d$ ion with a double occupancy (occupied by the doublon) and stands in what follows for the orbital degree of freedom. It is worth noting that the hopping (5) does not change the orbital flavor; thus, we expect that the resulting Hamiltonian is diagonal in the orbital degrees of freedom with only $D_{2}^{(\gamma)}$ operators.

Following the standard second-order perturbation expansion for spin-orbital systems [12], we can write the matrix elements of the low-energy exchange Hamiltonian $\mathcal{H}_{J}^{(\gamma)}(i, j)$ for a bond $\langle 12\rangle \| \gamma$ along the $\gamma$ axis as follows:

$$
\begin{aligned}
\left\langle e_{1}^{k}, e_{2}^{l}\left|\mathcal{H}_{J}^{(\gamma)}(1,2)\right| e_{1}^{k^{\prime}}, e_{2}^{l^{\prime}}\right\rangle & \\
= & -\sum_{n_{1}, n_{2}} \frac{1}{\varepsilon_{n 1}+\varepsilon_{n 2}} \\
& \times\left\langle e_{1}^{k}, e_{2}^{l}\left|H_{t}(1,2)\right| n_{1}, n_{2}\right\rangle \times\left\langle n_{1}, n_{2}\left|H_{t}(1,2)\right| e_{1}^{k^{\prime}}, e_{2}^{l^{\prime}}\right\rangle,
\end{aligned}
$$

with $\varepsilon_{n m}=E_{n, m}-E_{0, m}$ being the excitation energies for atoms at site $m=1,2$ with respect to the unperturbed ground state. The superexchange Hamiltonian $\mathcal{H}_{J}^{(\gamma)}(1,2)$ for a bond along $\gamma$ can be expressed in a matrix form by a $36 \times 36$ matrix, with dependence on $U_{m}, J_{m}^{H}$, and $I_{e}$ elements. There are two types of charge excitations: (i) the $d_{1}^{3} d_{2}^{4} \leftrightharpoons d_{1}^{4} d_{2}^{3}$ one, which creates a doublon at the $3 d$ impurity, and (ii) the $d_{1}^{3} d_{2}^{4} \leftrightharpoons d_{1}^{2} d_{2}^{5}$ one, which adds another doublon at the $4 d$ host site in the intermediate state. The second type of excitations involves more doubly occupied orbitals and has much larger excitation energy. It is therefore only a small correction to the leading term (i), as we discuss in Appendix A.

Similarly to the case of doped manganites [48], the dominant contribution to the effective low-energy spin-orbital Hamiltonian for the $3 d-4 d$ bond stems from the $d_{1}^{3} d_{2}^{4} \leftrightharpoons d_{1}^{4} d_{2}^{3}$ charge excitations, as they do not involve an extra double occupancy and the Coulomb energy $U_{2}$. The $3 d_{1}^{3} 4 d_{2}^{4} \leftrightharpoons 3 d_{1}^{4} 4 d_{2}^{3}$ charge excitations can be analyzed in a similar way as the $3 d_{i}^{3} 3 d_{j}^{4} \leftrightharpoons 3 d_{i}^{4} 3 d_{j}^{3}$ ones for an $\langle i j\rangle$ bond in doped manganites [48]. In both cases, the total number of doubly occupied orbitals does not change, so the main contributions come due to Hund's exchange. In the present case, one more parameter plays a role: 


$$
\Delta=I_{e}+3\left(U_{1}-U_{2}\right)-4\left(J_{1}^{H}-J_{2}^{H}\right),
$$

which stands for the mismatch potential energy (7) renormalized by the on-site Coulomb interactions $\left\{U_{m}\right\}$ and by Hund's exchange $\left\{J_{m}^{H}\right\}$. On a general ground, we expect $\Delta$ to be a positive quantity, since the repulsion $U_{m}$ should be larger for smaller $3 d$ shells than for the $4 d$ ones and $U_{m}$ is the largest energy scale in the problem.

Let us have a closer view on this dominant contribution of the effective low-energy spin-orbital Hamiltonian for the $3 d-4 d$ bond, given by Eq. (A2). For the analysis performed below and the clarity of our presentation, it is convenient to introduce some scaled parameters related to the interactions within the host and between the host and the impurity. For this purpose, we employ the exchange couplings $J_{\text {imp }}$ and $J_{\text {host }}$,

$$
\begin{aligned}
& J_{\text {imp }}=\frac{t^{2}}{4 \Delta}, \\
& J_{\text {host }}=\frac{4 t_{h}^{2}}{U_{2}},
\end{aligned}
$$

which follow from the virtual charge excitations generated by the kinetic energy; see Eqs. (2) and (5). We use their ratio to investigate the influence of the impurity on the spinorbital order in the host. Here, $t_{h}$ is the hopping amplitude between two $t_{2 g}$ orbitals at NN $4 d$ atoms, $J_{2}^{H}$ and $U_{2}$ refer to the host, and $\Delta$ (11) is the renormalized ionization energy of the $3 d-4 d$ bonds. The results depend as well on Hund's exchange element for the impurity and on the one at host atoms

$$
\begin{aligned}
& \eta_{\text {imp }}=\frac{J_{1}^{H}}{\Delta}, \\
& \eta_{\text {host }}=\frac{J_{2}^{H}}{U_{2}} .
\end{aligned}
$$

Note that the ratio introduced for the impurity $\eta_{\text {imp }}$ (14) has here a different meaning from Hund's exchange used here for the host $\eta_{\text {host }}(15)$, which cannot be too large by construction, i.e., $\eta_{\text {host }}<1 / 3$.

With the parametrization introduced above, the dominant term in the impurity-host Hamiltonian for the impurity spin $\vec{S}_{i}$ interacting with the neighboring host spins $\left\{\vec{S}_{j}\right\}$ at $j \in \mathcal{N}(i)$, deduced from $\mathcal{H}_{3 d-4 d}^{(\gamma)}(1,2)$ Eq. (A2), can be written in a rather compact form as follows:

$$
\mathcal{H}_{3 d-4 d}(i) \simeq \sum_{\gamma, j \in \mathcal{N}(i)}\left\{J_{S}\left(D_{j}^{(\gamma)}\right)\left(\vec{S}_{i} \cdot \vec{S}_{j}\right)+E_{D} D_{j}^{(\gamma)}\right\},
$$

where the orbital- (doublon-) dependent spin couplings $J_{S}\left(D_{j}^{(\gamma)}\right)$ and the doublon energy $E_{D}$ depend on $\eta_{\text {imp }}$. The evolution of the exchange couplings is shown in

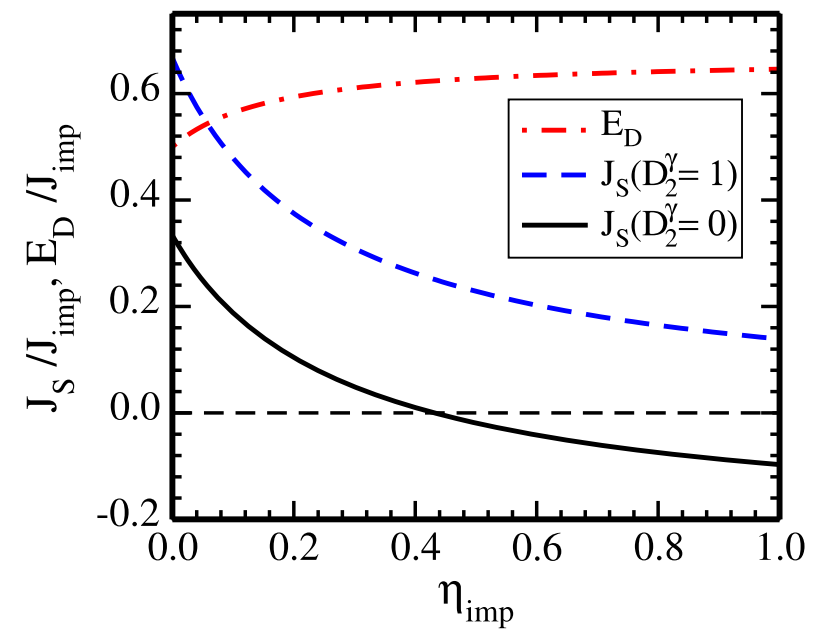

FIG. 4. Evolution of the spin exchange $J_{S}\left(D_{2}^{(\gamma)}\right)$ and the doublon energy $E_{D}$, both given in Eq. (16) for increasing Hund's exchange $\eta_{\text {imp }}$ at the impurity.

Fig. 4. We note that the dominant energy scale is $E_{D}^{\gamma}$, so for a single $3 d-4 d$ bond, the doublon will avoid occupying the inactive $(\gamma)$ orbital and the spins will couple with $J_{S}\left(D_{j}^{(\gamma)}=0\right)$, which can be either AF if $\eta_{\text {imp }} \lesssim 0.43$ or FM if $\eta_{\text {imp }}>0.43$. Thus, the spins at $\eta_{\text {imp }}=\eta_{\text {imp }}^{c} \simeq 0.43$ will decouple according to the $\mathcal{H}_{J}^{(\gamma)}(i, j)$ exchange.

Let us conclude this section by writing the complete superexchange Hamiltonian

$$
\mathcal{H}=\mathcal{H}_{3 d-4 d}+\mathcal{H}_{4 d-4 d}+\mathcal{H}_{\mathrm{SO}}
$$

where $\mathcal{H}_{3 d-4 d} \equiv \sum_{i} \mathcal{H}_{3 d-4 d}(i)$ includes all the $3 d-4 d$ bonds around impurities, $\mathcal{H}_{4 d-4 d}$ stands for the effective spin-orbital Hamiltonian for the $4 d$ host bonds, and $\mathcal{H}_{\mathrm{SO}}$ is the spin-orbit interaction in the host. The former term we explain below, while the latter one is defined in Sec. V B, where we analyze the quantum corrections and the consequences of spin-orbit interaction. The superexchange in the host for the bonds $\langle i j\rangle$ along the $\gamma=a, b$ axes [81]

$$
\mathcal{H}_{4 d-4 d}=J_{\text {host }} \sum_{\langle i j\rangle \| \gamma}\left\{J_{i j}^{(\gamma)}\left(\vec{S}_{i} \cdot \vec{S}_{j}+1\right)+K_{i j}^{(\gamma)}\right\}
$$

depends on $J_{i j}^{(\gamma)}$ and $K_{i j}^{(\gamma)}$ operators acting only in the orbital space. They are expressed in terms of the pseudospin operators defined in the orbital subspace spanned by the two orbital flavors active along a given direction $\gamma$, i.e.,

$$
\begin{aligned}
J_{i j}^{(\gamma)}= & \frac{1}{2}\left(2 r_{1}+1\right)\left(\vec{\tau}_{i} \cdot \vec{\tau}_{j}\right)^{(\gamma)}-\frac{1}{2} r_{2}\left(\tau_{i}^{z} \tau_{j}^{z}\right)^{(\gamma)} \\
& +\frac{1}{8}\left(n_{i} n_{j}\right)^{(\gamma)}\left(2 r_{1}-r_{2}+1\right)-\frac{1}{4} r_{1}\left(n_{i}+n_{j}\right)^{(\gamma)},
\end{aligned}
$$




$$
\begin{aligned}
K_{i j}^{(\gamma)}= & r_{1}\left(\vec{\tau}_{i} \cdot \vec{\tau}_{j}\right)^{(\gamma)}-r_{2}\left(\tau_{i}^{z} \tau_{j}^{z}\right)^{(\gamma)}+\frac{1}{4}\left(r_{1}+r_{2}\right)\left(n_{i} n_{j}\right)^{(\gamma)} \\
& -\frac{1}{4}\left(r_{1}+1\right)\left(n_{i}+n_{j}\right)^{(\gamma)}
\end{aligned}
$$

with

$$
r_{1}=\frac{\eta_{\text {host }}}{1-3 \eta_{\text {host }}}, \quad r_{2}=\frac{\eta_{\text {host }}}{1+2 \eta_{\text {host }}},
$$

standing for the multiplet structure in charge excitations, and the orbital operators $\left\{\vec{\tau}_{i}^{(\gamma)}, n_{i}^{(\gamma)}\right\}$ that for the $\gamma=c$ axis take the form

$$
\begin{gathered}
\vec{\tau}_{i}^{(c)}=\frac{1}{2}\left(\begin{array}{cc}
a_{i}^{\dagger} & b_{i}^{\dagger}
\end{array}\right) \cdot \vec{\sigma} \cdot\left(\begin{array}{ll}
a_{i} & b_{i}
\end{array}\right)^{\top}, \\
n_{i}^{(c)}=a_{i}^{\dagger} a_{i}+b_{i}^{\dagger} b_{i} .
\end{gathered}
$$

For the directions $\gamma=a, b$ in the considered $(a, b)$ plane, one finds equivalent expressions by cyclic permutation of the axis labels $\{a, b, c\}$ in the above formulas. This problem is isomorphic with the spin-orbital superexchange in the vanadium perovskites [50,51], where a hole in the $\{a, b\}$ doublet plays an equivalent role to the doublon in the present case. The operators $\left\{a_{i}^{\dagger}, b_{i}^{\dagger}, c_{i}^{\dagger}\right\}$ are the doublon- (hard-core boson-) creation operators in the orbital $\gamma=a, b, c$, respectively, and they satisfy the local constraint

$$
a_{i}^{\dagger} a_{i}+b_{i}^{\dagger} b_{i}+c_{i}^{\dagger} c_{i}=1,
$$

meaning that exactly one doublon (9) occupies one of the three $t_{2 g}$ orbitals at each site $i$. These bosonic occupation operators coincide with the previously used doublon occupation operators $D_{j}^{(\gamma)}$, i.e., $D_{j}^{(\gamma)}=\gamma_{j}^{\dagger} \gamma_{j}$ with $\gamma=a, b, c$. Below, we follow first the classical procedure to determine the ground states of single impurities in Sec. III and at macroscopic doping in Sec IV.

\section{SINGLE $3 d$ IMPURITY IN $4 d$ HOST}

\section{A. Classical treatment of the impurity problem}

In this section, we describe the methodology that we applied for the determination of the phase diagrams for a single impurity reported below in Sec. III C and next at macroscopic doping, as presented in Sec. IV. Let us consider first the case of a single $3 d$ impurity in the $4 d$ host. Since the interactions in the model Hamiltonian are only effective ones between NN sites, it is sufficient to study the modification of the spin-orbital order around the impurity for a given spin-orbital configuration of the host by investigating a cluster of $L=13$ sites shown in Fig. 5 . We assume the $C$-AF spin order (FM chains coupled antiferromagnetically) accompanied by $G$-AO order within

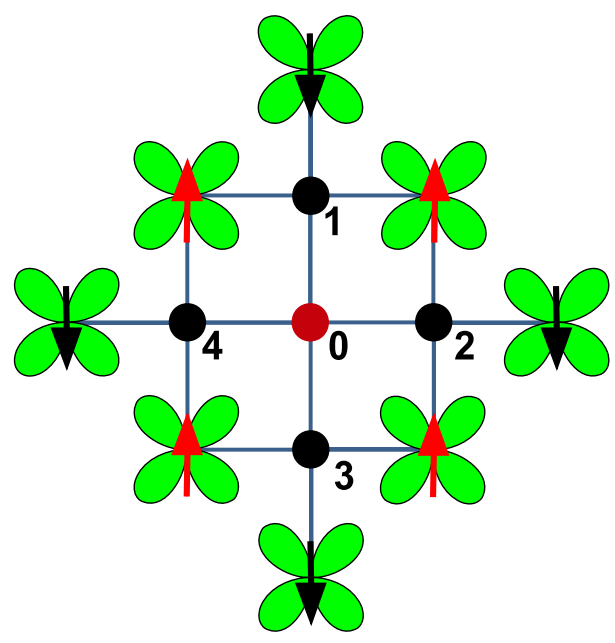

FIG. 5. Schematic top view of the cluster used to obtain the phase diagrams of the $3 d$ impurity within the $4 d$ host in an $(a, b)$ plane. The impurity is at the central site $i=0$ that belongs to the $c$-orbital host sublattice. For the outer sites in this cluster, the spin-orbital configuration is fixed and determined by the undoped $4 d$ host (with spins and $c$ orbitals shown here) having $C$-AF or $G$-AO order; see Fig. 2. For the central $i=0$ site, the spin state, and for the host sites $i=1, \ldots, 4$, the spin-orbital configurations are determined by minimizing the energy of the cluster.

the host, which is the spin-orbital order occurring for the realistic parameters of a $\mathrm{RuO}_{2}$ plane [81]; see Fig. 2. Such a spin-orbital pattern turns out to be the most relevant one when considering the competition between the host and the impurity as due to the $\mathrm{AO}$ order within the $(a, b)$ plane. Other possible configurations with uniform orbital order and $\mathrm{AF}$ spin pattern, e.g., $G$-AF order, will also be considered in the discussion throughout the manuscript. The sites $i=1,2,3,4$ inside the cluster in Fig. 5 have active spin and orbital degrees of freedom, while the impurity at site $i=0$ has only spin degrees of freedom. At the remaining sites, the spin-orbital configuration is assigned, following the order in the host, and it does not change along the computation.

To determine the ground state, we assume that the spinorbital degrees of freedom are treated as classical variables. This approximation implies that for the bonds between atoms in the host, we use the Hamiltonian (18) and neglect quantum fluctuations; i.e., in the spin sector, we keep only the $z$ th (Ising) spin components and in the orbital one only the terms that are proportional to the doublon occupation numbers (9) and to the identity operators. Similarly, for the impurity-host bonds, we use the Hamiltonian Eq. (16) by keeping only the $z$ th projections of spin operators. Since we do neglect the fluctuation of the spin amplitude, it is enough to consider only the maximal and minimal values of $\left\langle S_{i}^{z}\right\rangle$ for spin $S=3 / 2$ at the impurity sites and $S=1$ at the host atoms. With these assumptions, we can construct all the possible configurations by varying the spin and orbital configurations at the sites from $i=1$ to $i=4$ in the cluster shown in Fig. 5. Note that the outer ions in the cluster all 
belong to the same sublattice, so two distinct cases have to be considered to probe all the configurations. Since physically it is unlikely that a single impurity will change the orbital order of the host globally, thus we will not compare the energies from these two cases and analyze two classes of solutions separately; see Sec. III B. Then, the lowest energy configuration in each class provides the optimal spin-orbital pattern for the NNs around the $3 d$ impurity. In the case of degenerate classical states, the spin-orbital order is established by including quantum fluctuations.

In the case of a periodic doping analyzed in Sec. IV, we use a similar strategy in the computation. Taking the most general formulation, we employ larger clusters having both size and shape that depend on the impurity distribution and on the spin-orbital order in the host. For this purpose, the most natural choice is to search for the minimum energy configuration in the elementary unit cell that can reproduce the full lattice by a suitable choice of the translation vectors. Such analysis is computationally expensive but doable for a periodic distribution of the impurities that is commensurate to the lattice because it yields a unit cell of relatively small size for doping around $x=0.1$. Otherwise, for the incommensurate doping, the size of the unit cell can lead to a configuration space of a dimension that impedes finding of the ground state. This problem is computationally more demanding, and to avoid the comparison of all the energy configurations, we have employed the Metropolis algorithm at low temperature to achieve the optimal configuration iteratively along the convergence process. Note that this approach is fully classical, meaning that the spins of the host and impurity are treated as Ising variables and the orbital fluctuations in the host's Hamiltonian Eq. (18) are omitted. They will be addressed in Sec. VA.

\section{B. Two nonequivalent $3 d$ doping cases}

The single-impurity problem is the key case to start with because it shows how the short-range spin-orbital correlations are modified around the $3 d$ atom due to the host and host-impurity interactions in Eq. (17). The analysis is performed by fixing the strength between Hund's exchange and Coulomb interaction within the host (6) at $\eta_{\text {host }}=0.1$, and by allowing for a variation of the ratio between the host-impurity interaction (16) and the Coulomb coupling at the impurity site. The choice of $\eta_{\text {host }}=0.1$ is made here because this value is within the physically relevant range for the case of the ruthenium materials. Small variations of $\eta_{\text {host }}$ do not affect the obtained results qualitatively.

As we have already discussed in the model derivation, the sign of the magnetic exchange between the impurity and the host depends on the orbital occupation of the $4 d$ doublon around the $3 d$ impurity. The main aspect that controls the resulting magnetic configuration is then given by the character of the doublon orbitals around the impurity, depending on whether they are active or inactive along the considered $3 d-4 d$ bond. To explore such a competition quantitatively, we investigate $G$-AO order for the host with alternation of $a$ - and $c$-doublon configurations accompanied by the $C$-AF spin pattern; see Fig. 2. Note that the $a$ orbitals are active only along the $b$ axis, while the $c$ orbitals are active along both the $a$ and $b$ axes [80]. This state has the lowest energy for the host in a wide range of parameters for Hund's exchange, the Coulomb element, and the crystal-field potential [81].

Because of the specific orbital pattern of Fig. 2, the $3 d$ impurity can substitute one of two distinct $4 d$ sites that are considered separately below, either with the $a$ or with the $c$ orbital occupied by the doublon. Since the two $4 d$ atoms have nonequivalent surrounding orbitals, not always active along the $3 d-4 d$ bond, we expect that the resulting ground state will have a modified spin-orbital order. Indeed, if the $3 d$ atom replaces the $4 d$ one with the doublon in the $a$ orbital, then all the $4 d$ neighboring sites have active doublons along the connecting $3 d-4 d$ bonds because they are in the $c$ orbitals. On the contrary, the substitution at the $4 d$ site with $c$-orbital doublon configuration leads to an impurity state with its neighbors having both active and inactive doublons. Therefore, we do expect a more intricate competition with frustrated host-impurity interactions for the latter case of an impurity occupying the $4 d$ site with $c$-orbital configuration, as we show in Sec. III D.

\section{Doping removing a doublon in an $a$ orbital}

We start by considering the physical situation where the $3 d$ impurity replaces a $4 d$ ion with the doublon within the $a$ orbital. The ground-state phase diagram and the schematic view of the spin-orbital pattern are reported in Fig. 6 in terms of the ratio $J_{\text {imp }} / J_{\text {host }}(14)$ and the strength of Hund's exchange coupling $\eta_{\text {imp }}(12)$ at the $3 d$ site. There are three different ground states that appear in the phase diagram. Taking into account the structure of the $3 d-4 d$ spinorbital exchange (16), we expect that, in the regime where the host-impurity interaction is greater than that in the host, the $4 d$ neighbors to the impurity tend to favor the spinorbital configuration set by the $3 d-4 d$ exchange. In this case, since the orbitals surrounding the $3 d$ site already minimize the $3 d-4 d$ Hamiltonian, we expect that the optimal spin configuration corresponds to the $4 d$ spins aligned either antiferromagnetically or ferromagnetically with respect to the impurity $3 d$ spin.

The neighbor spins are AF to the $3 d$ spin impurity in the $\mathrm{AF} a$ phase, while the FM $a$ phase is just obtained from $\mathrm{AF} a$ by reversing the spin at the impurity and having all the $3 d-4 d$ bonds FM. It is interesting to note that due to the host-impurity interaction, the $C$-AF spin pattern of the host is modified in both the $\mathrm{AF} a$ and the FM $a$ ground states. Another intermediate configuration that emerges when the host-impurity exchange is weak is the intermediate frustrated spin at $a$ site (FSa) phase where the impurity spin is undetermined and its configuration in the initial $C$-AF phase is degenerate with the one obtained after the spin-inversion 

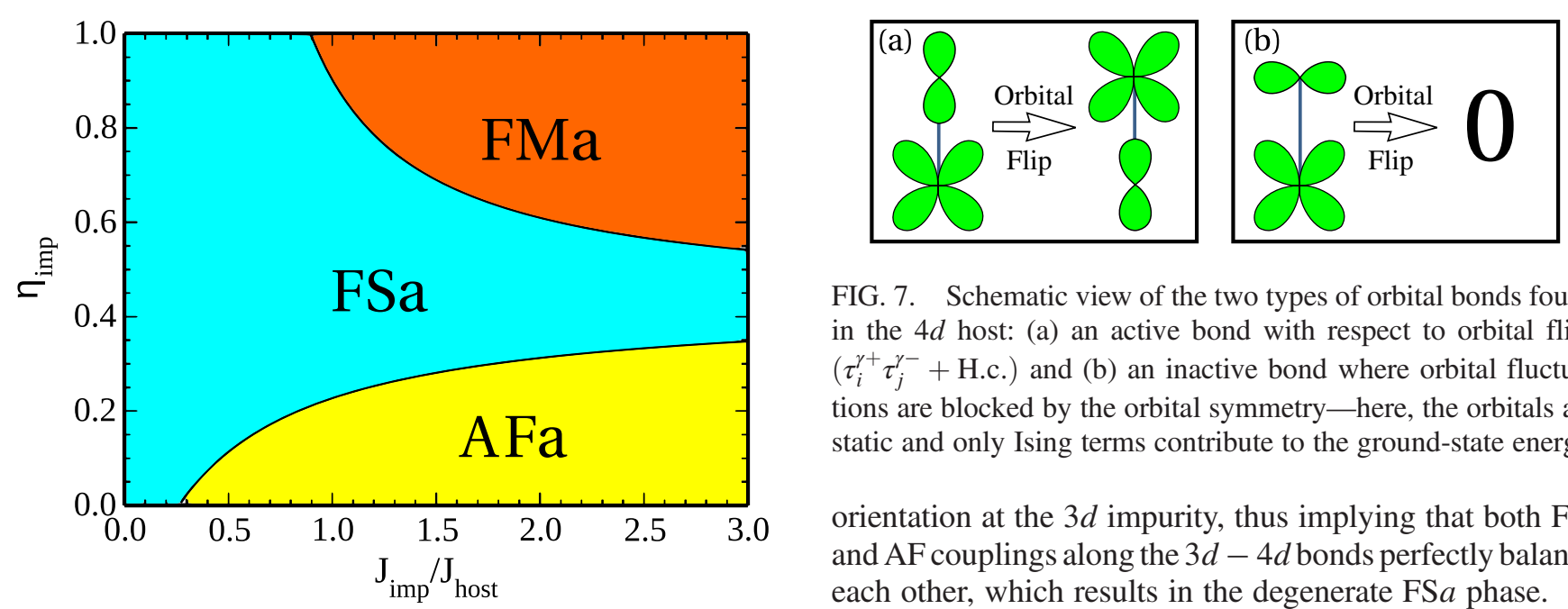

FIG. 7. Schematic view of the two types of orbital bonds found in the $4 d$ host: (a) an active bond with respect to orbital flips $\left(\tau_{i}^{\gamma+} \tau_{j}^{\gamma-}+\right.$ H.c. $)$ and (b) an inactive bond where orbital fluctuations are blocked by the orbital symmetry-here, the orbitals are static and only Ising terms contribute to the ground-state energy.

orientation at the $3 d$ impurity, thus implying that both FM and AF couplings along the $3 d-4 d$ bonds perfectly balance each other, which results in the degenerate FS $a$ phase.

It is worth pointing out that there is a quite large region of the phase diagram where the FS $a$ state is stabilized and the spin-orbital order of the host is not affected by doping with the possibility of having large degeneracy in the spin configuration of the impurities. On the other hand, by inspecting the $c$ orbitals around the impurity (Fig. 6) from the point of view of the full host's Hamiltonian Eq. (18) with orbital flips included $\left(\tau_{i}^{\gamma+} \tau_{j}^{\gamma-}+\right.$ H.c. $)$, one can easily find out that the frustration of the impurity spin can be released by quantum orbital fluctuations. Note that the $c$ orbitals around the impurity in the $a(b)$ direction have quite different surroundings. The ones along the $a$ axis are connected by two active bonds along the $b$ axis with orbitals $a$, as in Fig. 7(a), while the ones along $b$ are connected with only one active $a$ orbital along the same $b$ axis. Therefore in the perturbative expansion, the orbital flips will contribute only along the $b$ bonds (for the present $G$-AO order) and admix the $a$-orbital character to $c$ orbitals along them, while such processes will be blocked for the bonds along the $a$ axis, as also for $b$ orbitals along the $b$ axis; see Fig. 7(b).

This fundamental difference can easily be included in the host-impurity bond in the mean-field manner by setting $\left\langle D_{i \pm b, \gamma}\right\rangle=0$ for the bonds along the $b$ axis and $0<\left\langle D_{i \pm a, \gamma}\right\rangle<1$ for the bonds along the $a$ axis. Then, one can easily check that for the impurity spin pointing downward, we get the energy contribution from the spin-spin bonds, which is given by $E_{\downarrow}=\alpha\left(\eta_{\text {host }}\right)\left\langle D_{i \pm a, \gamma}\right\rangle$, and for the impurity spin pointing upward, we have $E_{\uparrow}=-\alpha\left(\eta_{\text {host }}\right)\left\langle D_{i \pm a, \gamma}\right\rangle$, with $\alpha\left(\eta_{\text {host }}\right)>0$. Thus, it is clear that any admixture of the virtual orbital flips in the host's wave function polarizes the impurity spin upward so that the $C$-AF order of the host will be restored.

\section{Doping removing a doublon in a $c$ orbital}

Let us move to the case of the $3 d$ atom replacing the doublon at the $c$ orbital. As anticipated above, this configuration is more intricate because the orbitals surrounding the impurity, as originated by the $C$-AF or $\mathrm{AO}$ order within the host, lead to nonequivalent $3 d-4 d$ bonds. 

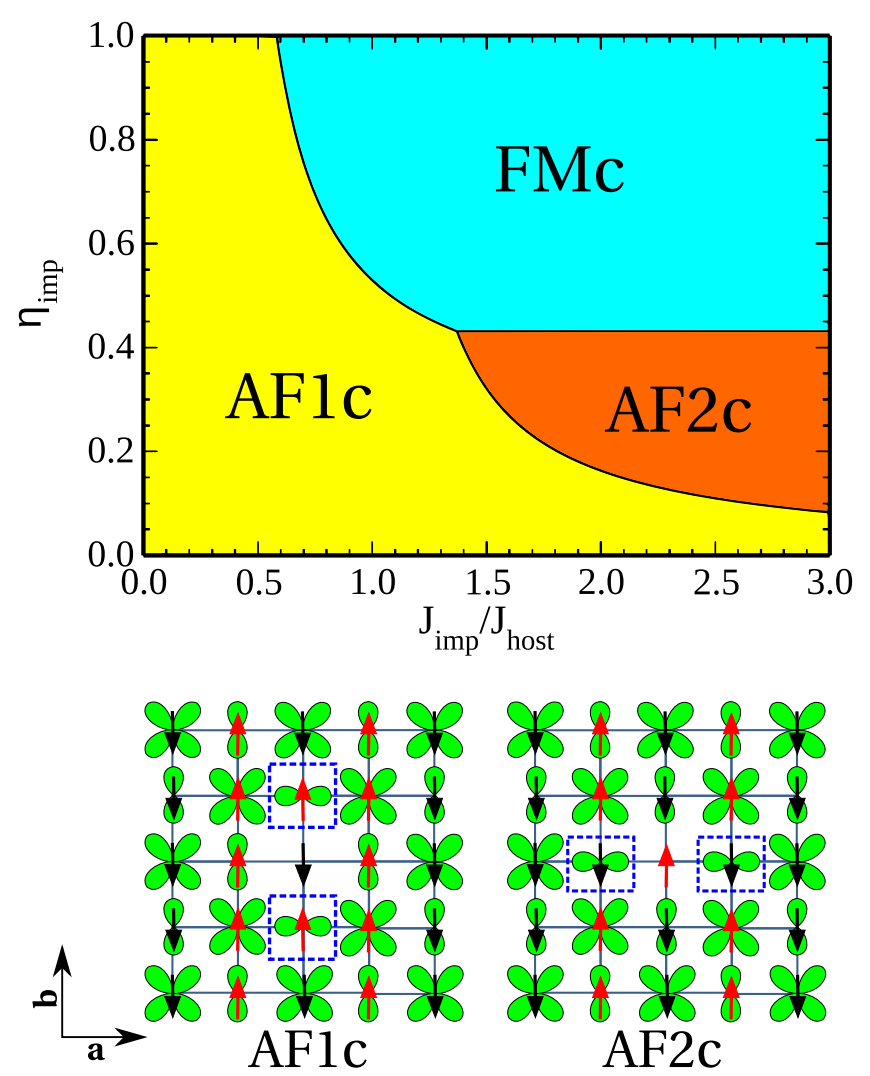

FIG. 8. Top: Phase diagram of the $3 d$ impurity in the $4 d$ host with $C$-AF or $G$-AO order and the impurity doped at the $c$-doublon sublattice. Different colors refer to local spin order around the impurity: $\mathrm{AF} c 1, \mathrm{AF} c 2$, and FM. Bottom: Schematic view of spin-orbital patterns for the two $\mathrm{AF}$ ground-state configurations shown in the top panel; the FMc phase differs from the $\mathrm{AF} c 2$ one only by spin inversion at the $3 d$ atom. The $3 d$ atom is at the central site and has no doublon orbital; the frames highlight the spin-orbital defects caused by the impurity. As in Fig. 6, the labels AF and FM refer to the impurity-spin orientation with respect to the neighboring $4 d$ sites.

There are two bonds with the doublon occupying an inactive orbital (and having no hybridization with the $t_{2 g}$ orbitals at the $3 d$ atom) and two remaining bonds with doublons in active $t_{2 g}$ orbitals.

Since the $3 d-4 d$ spin-orbital exchange depends on the orbital polarization of $4 d$ sites, we do expect a competition that may modify significantly the spin-orbital correlations in the host. Indeed, one observes that three configurations compete, denoted as $\mathrm{AF} 1 c$, $\mathrm{AF} 2 c$, and FMc; see Fig. 8. In the regime where the host-impurity exchange dominates, the system tends to minimize the energy due to the $3 d-4 d$ spin-orbital coupling, and thus, the orbitals become polarized in the active configurations compatible with the $C$-AF or $G$-AO pattern, and the host-impurity spin coupling is AF for $\eta_{\text {imp }} \leq 0.43$ while it is FM otherwise. This region resembles orbital polarons in doped manganites $[39,42]$. Also, in this case, the orbital polarons arise because they minimize the double-exchange energy [46].

On the contrary, for weak spin-orbital coupling between the impurity and the host, there is an interesting cooperation between the $3 d$ and $4 d$ atoms. Since the strength of the impurity-host coupling is not sufficient to polarize the orbitals at the $4 d$ sites, it is preferable to have an orbital rearrangement to the configuration with inactive orbitals on $3 d-4 d$ bonds and spin flips at $4 d$ sites. In this way, the spin-orbital exchange is optimized in the host and also on the $3 d-4 d$ bonds. The resulting state has an AF coupling between the host and the impurity as it should when all the orbitals surrounding the $3 d$ atoms are inactive with respect to the bond direction. This modification of the orbital configuration induces the change in spin orientation. The double-exchange bonds (with inactive doublon orbitals) along the $b$ axis are then blocked and the total energy is lowered, in spite of the frustrated spin-orbital exchange in the host. As a result, in the AF1c state, the spins surrounding the impurity are aligned and antiparallel to the spin at the $3 d$ site.

Concerning the host $C$-AF or $G$-AO order, we note that it is modified only along the direction where the FM correlations develop and spin defects occur within the chain doped by the $3 d$ atom. The FM order is locally disturbed by the $3 d$ defect antiferromagnetically coupled spins surrounding it. Note that this phase is driven by the orbital vacancy as the host develops more favorable orbital bonds to gain the energy in the absence of the orbital degrees of freedom at the impurity. At the same time, the impurity-host bonds do not generate too big energy losses as (i) either $\eta_{\text {imp }}$ is so small that the loss due to $E_{D}$ is compensated by the gain from the superexchange $\propto$ $J_{S}\left(D_{j}^{(\gamma)}=1\right.$ ) (all these bonds are AF) (see Fig. 4) or (ii) $J_{\text {imp }} / J_{\text {host }}$ is small, meaning that the overall energy scale of the impurity-host exchange remains small. Interestingly, if we compare the $\mathrm{AF} 1 c$ with the $\mathrm{AF} 2 c$ ground states, we observe that the disruption of the $C$-AF or $G$-AO order is anisotropic and occurs either along the FM chains in the AF1c phase or perpendicular to the FM chains in the $\mathrm{AF} 2 c$ phase. No spin frustration is found here, in contrast to the FSa phase in the case of $a$-doublon doping; see Fig. 6.

Finally, we point out that a very similar phase diagram can be obtained assuming that the host has the FM or $G$-AO order with $a$ and $b$ orbitals alternating from site to site. Such a configuration can be stabilized by a distortion that favors the out-of-plane orbitals. In this case, there is no difference in doping at one or the other sublattice. The main difference is found in energy scales-for the $G$-AF or $C$-AO order, the diagram is similar to the one of Fig. 8 if we rescale $J_{\text {imp }}$ by half, which means that the $G$-AF order is softer than the $C$-AF one. Note also that in the peculiar $\mathrm{AF} 1 c$ phase, the impurity does not induce any changes in the host for the FM or AO ordered host. Thus, we can safely conclude that the observed change in the orbital order for 
the $C$-AF host in the AF1c phase is due to the presence of the $c$ orbitals, which are not directional in the $(a, b)$ plane.

Summarizing, we have shown the complexity of local spin-orbital order around $t_{2 g}^{3}$ impurities in a $4 t_{2 g}^{4}$ host. It is remarkable that such impurity spins not only modify the spinorbital order around them in a broad regime of parameters but also are frequently frustrated. Hence, the behavior of the impurity highlights the importance of quantum effects beyond the present classical approach that release frustration, as we show in Sec. VA.

\section{PERIODIC $3 d$ DOPING IN A $4 d$ HOST}

\section{A. General remarks on finite doping}

In this section, we analyze the spin-orbital patterns due to a finite concentration $x$ of $3 d$ impurities within the $4 d$ host with $C$-AF or $G$-AO order, assuming that the $3 d$ impurities are distributed in a periodic way. The study is performed for three representative doping distributions - the first one $x=1 / 8$ is commensurate with the underlying spin-orbital order and the other two are incommensurate with respect to it, meaning that in such cases, doping at both the $a$ - and $c$-doublon sites is imposed simultaneously.

As the impurities lead to local energy gains due to the $3 d-4 d$ bonds surrounding them, we expect that the most favorable situation is when they are isolated and have maximal distances between one another. Therefore, we select the largest possible distances for the three doping levels used in our study: $x=1 / 8, x=1 / 5$, and $x=1 / 9$. This choice allows us to cover different regimes of competition between the spin-orbital coupling within the host and the $3 d-4 d$ coupling. While single impurities may only change spin-orbital order locally, we use here a high enough doping to investigate possible global changes in spin-orbital order, i.e., whether they can occur in the respective parameter regime. The analysis is performed as for a single impurity, by assuming the classical spin and orbital variables and by determining the configuration with the lowest energy. For this analysis, we set the spatial distribution of the $3 d$ atoms and we determine the spin and orbital profile that minimizes the energy.

\section{B. $C$-AF phase with $x=1 / 8$ doping}

We begin with the phase diagram obtained at $x=1 / 83 d$ doping; see Fig. 9. In the regime of strong impurity-host coupling, the $3 d-4 d$ spin-orbital exchange determines the orbital and spin configuration of the $4 d$ atoms around the impurity. The most favorable state is when the doublon occupies $c$ orbitals at the NN sites to the impurity. The spin correlations between the impurity and the host are AF (FM), if the amplitude of $\eta_{\text {imp }}$ is below (above) $\eta_{\text {imp }}^{c}$, leading to the AF $a$ and FM $a$ states; see Fig. 9. The AF $a$ state has a stripelike profile with AF chains alternated by FM domains (consisting of three chains) along the diagonal of the square lattice. Even if the coupling between the impurity and the

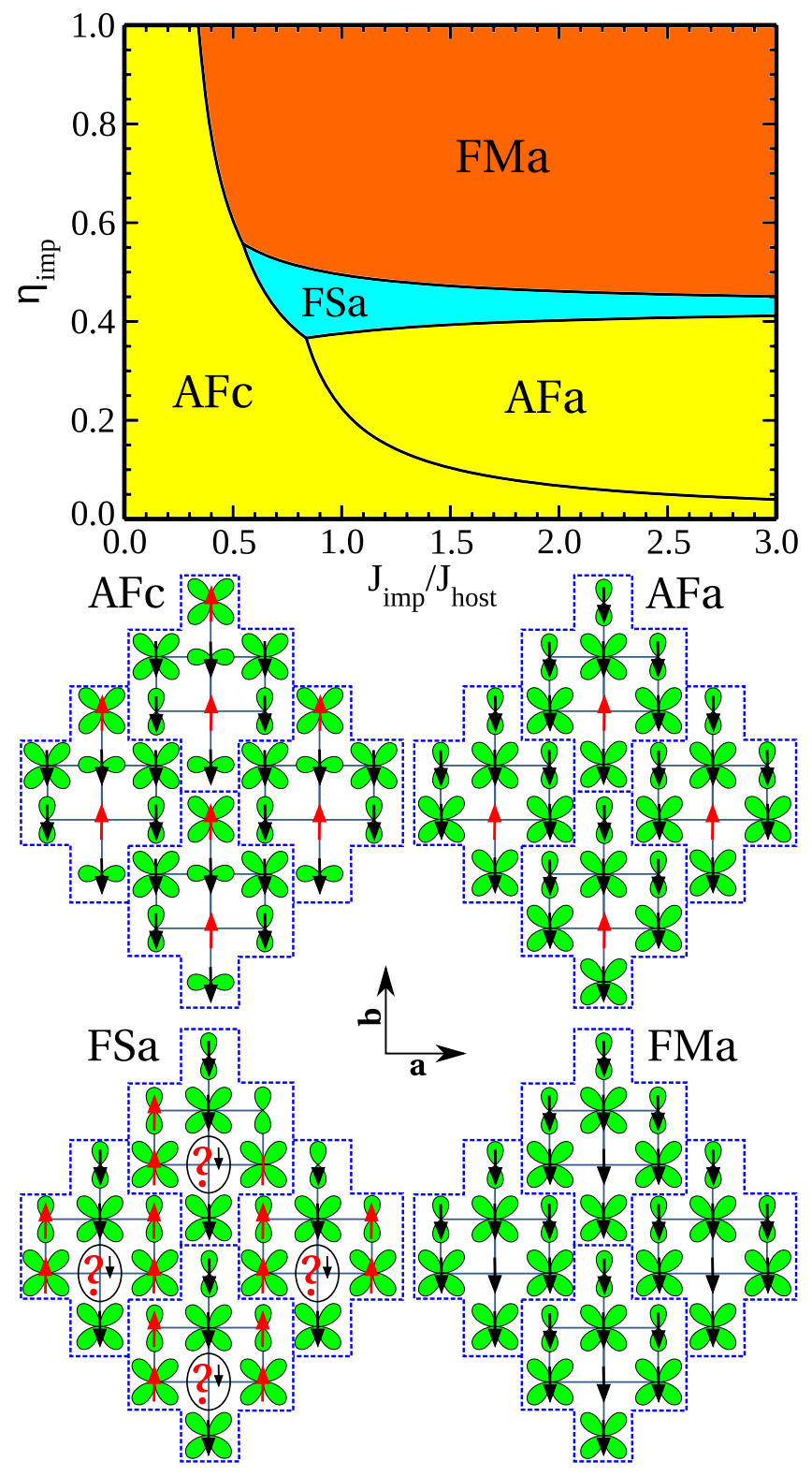

FIG. 9. Top panel: Ground-state diagram obtained for periodic $3 d$ doping $x=1 / 8$. Different colors refer to local spin order around the impurity: $\mathrm{AF} a, \mathrm{AF} c, \mathrm{FS} a$, and $\mathrm{FM} a$. Bottom panel: Schematic view of the ground-state configurations within the four eight-site unit cells (indicated by dashed blue lines) for the phases shown in the phase diagram. The question marks in the FS $a$ phase indicate frustrated impurity spins within the classical approach - the spin direction (small arrows) is fixed only by quantum fluctuations. The $3 d$ atoms are placed at the sites where orbitals are absent.

host is $\mathrm{AF}$ for all the bonds in the $\mathrm{AF} a$ state, the overall configuration has a residual magnetic moment originating by the uncompensated spins and by the cooperation between the spin-orbital exchange in the $4 d$ host and that for the $3 d-4 d$ bonds. Interestingly, at the point where the dominant $3 d-4 d$ exchange tends to 0 (i.e., for $\eta_{\text {imp }} \simeq \eta_{\text {imp }}^{c}$ ), one finds a region of the FS $a$ phase that is analogous to the FSa phase found in Sec. III C for a single 
impurity; see Fig. 6. Again, the impurity spin is frustrated in a purely classical approach, but this frustration is easily released by the orbital fluctuations in the host so that the $C$-AF order of the host can be restored. This state is stable for the amplitude of $\eta_{\text {imp }}$ being close to $\eta_{\text {imp }}^{c}$.

The regime of a small $J_{\text {imp }} / J_{\text {host }}$ ratio is qualitatively different-an orbital rearrangement around the impurity takes place, with a preference to move the doublons into the inactive orbitals along the $3 d-4 d$ bonds. Such orbital configurations favor the AF spin coupling at all the $3 d-4 d$ bonds, which is stabilized by the $4 d-4 d$ superexchange [38]. This configuration is peculiar because it generally breaks inversion and does not have any plane of mirror symmetry. It is worth pointing out that the original order in the $4 d$ host is completely modified by the small concentration of $3 d$ ions, and one finds that the AF coupling between the $3 d$ impurity and the $4 d$ host generally leads to patterns such as the $\mathrm{AF} c$ phase where $\mathrm{FM}$ chains alternate with $\mathrm{AF}$ ones in the $(a, b)$ plane. Another relevant issue is that the cooperation between the host and impurity can lead to a fully polarized FM $a$ state which implies that doping can release the orbital frustration that was present in the host with the $C$-AF or $G$-AO order.

\section{Phase diagram for periodic $x=1 / 5$ doping}

Next, we consider doping $x=1 / 5$ with a given periodic spatial profile that concerns both doublon sublattices. We investigate the $3 d$ spin impurities separated by the translation vectors $\vec{u}=(i, j)$ and $\vec{v}=(2,-1)$ (one can show that for general periodic doping $\left.x,|\vec{u}|^{2}=x^{-1}\right)$, so there is a mismatch between the impurity periodicity and the twosublattice $G-\mathrm{AO}$ order in the host. One finds that the present case (see Fig. 10) has a similar general structure of

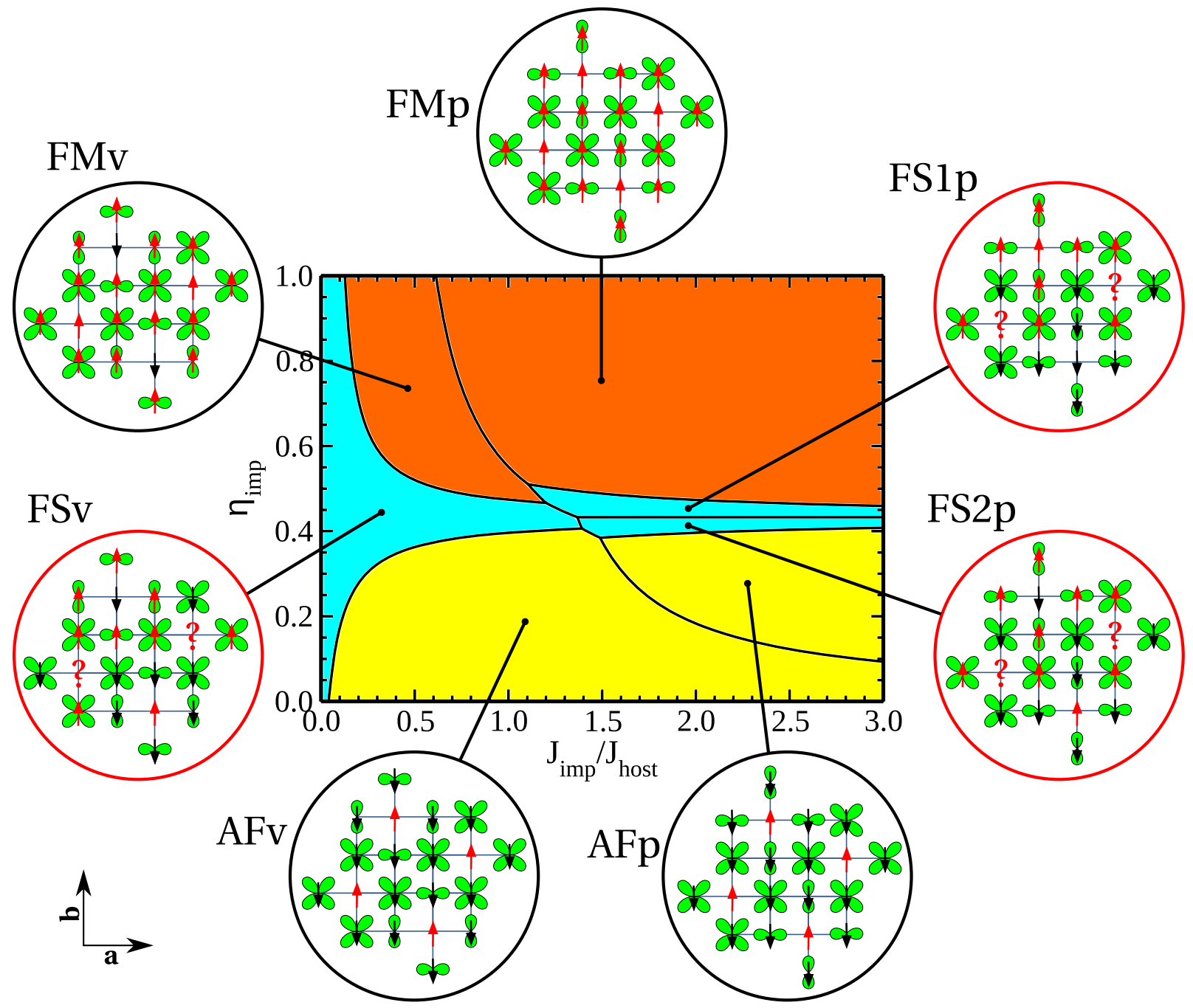

FIG. 10. Ground-state diagram for an $x=1 / 5$ periodic concentration of $3 d$ impurities (sites where orbitals are absent) with schematic views of the ground-state configurations obtained for the unit cell of 20 sites. The spin and orbital orders are shown by arrows and orbitals occupied by doublons; magnetic phases (AF, FS, and FM) are highlighted by different colors. The question marks in the FS states (red circles) indicate frustrated impurity spins within the classical approach. 
the phase diagram to the case of $x=1 / 8$ (Fig. 9), with AF correlations dominating for $\eta_{\text {imp }}$ lower than $\eta_{\text {imp }}^{c}$ and FM ones otherwise. Because of the specific doping distribution, there are more phases appearing in the groundstate phase diagram. For $\eta_{\text {imp }}<\eta_{\text {imp }}^{c}$, the most stable spin configuration is with the impurity coupled antiferromagnetically to the host as it happens both in the AF vacancy $(\mathrm{AF} v)$ and the $\mathrm{AF}$ polaronic $(\mathrm{AF} p)$ ground states. The difference between the two AF states arises due to the orbital arrangement around the impurity. For the weak ratio of the impurity to the host spin-orbital exchange $J_{\text {imp }} / J_{\text {host }}$, the orbitals around the impurity are all inactive ones. On the contrary, in the strong impurity-host-coupling regime, all the orbitals are polarized to be in active (polaronic) states around the impurity. Both states have been found as the $\mathrm{AF} 1 c$ and $\mathrm{AF} 2 c$ phases in the single-impurity problem (Fig. 8).

More generally, for all phases, the boundary given by an approximate hyperbolic relation $\eta_{\text {imp }} \propto J_{\text {imp }}^{-1}$ separates the phases where the orbitals around impurities in the $c$-orbital sublattice are all inactive (small $\eta_{\text {imp }}$ ) from those where all the orbitals are active (large $\eta_{\text {imp }}$ ). The inactive orbital around the impurity always stabilizes the AF coupling between the impurity spin and host spins, whereas the active orbitals can give either AF or FM exchange depending on $\eta_{\text {imp }}$ (hence, $\eta_{\text {imp }}^{c}$; see Fig. 4). Since the doping does not match the size of the elementary unit cell, the resulting ground states do not exhibit specific symmetries in the spin-orbital pattern. They are generally FM due to the uncompensated magnetic moments, and the impurity feels screening by the presence of the surrounding host spins being antiparallel to the impurity spin.

By increasing Hund's exchange coupling at the $3 d$ ion, the system develops a fully FM state in a large region of the ground-state diagram due to the possibility of suitable orbital polarization around the impurity. On the other hand, in the limit where the impurity-host bonds are weak, so either for $\eta_{\text {imp }} \simeq \eta_{\text {imp }}^{c}$ and large enough $J_{\text {imp }} / J_{\text {host }}$ so that all orbitals around the impurity are active, or just for small $J_{\text {imp }} / J_{\text {host }}$, we get the FS phases where the impurity spin at the $a$-orbital sublattice is undetermined in the present classical approach. A similar situation occurred in the FS $a$ phase of a single-impurity problem and at $x=1 / 8$ periodic doping (see Figs. 6 and 9), but there it was still possible to identify the favored impurity-spin polarization by considering the orbital flips in the host around the impurity.

However, the situation here is different, as the host's order is completely altered by doping and has became isotropic, in contrast to the initial $C$-AF order (Fig. 2), which breaks the planar symmetry between the $a$ and $b$ directions. It is precisely this symmetry breaking that favors one impurity-spin polarization over the other one. Here, this mechanism is absent-one can easily check

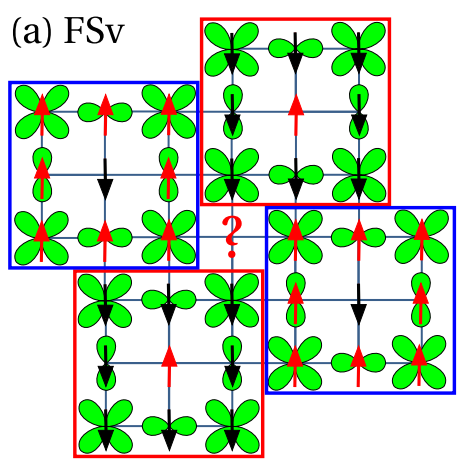

(b) FS1p, FS2p

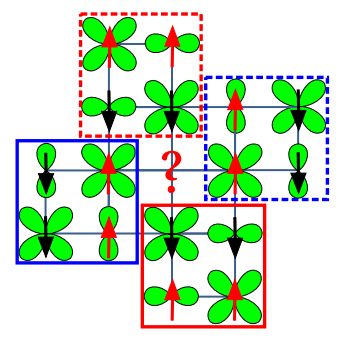

FIG. 11. Isotropic surrounding of the degenerate impurity spins in the FS $v$ and $\mathrm{FS} p$ phases in the case of $x=1 / 5$ periodic doping (Fig. 10). Frames mark the clusters that are not connected with orbitally active bonds.

that the neighborhood of the $c$ orbitals surrounding impurities is completely equivalent in both directions (see Fig. 11 for the view of these surroundings) so that the orbital flip argument is no longer applicable. In such a peculiar situation that occurs in the classical approach we indicate frustration in the spin direction by question marks in Fig. 10.

In Fig. 11, we can see that in both FS vacancy (FSv) and FS polaronic (FSp) phases, the orbitals are grouped in $3 \times 3$ clusters and $2 \times 2$ plaquettes, respectively, that encircle the degenerate impurity spins. For the FS $v$ phase, we can distinguish between two kinds of plaquettes with nonzero spin polarization differing by a global spin inversion. In the case of FS phases, we observe four plaquettes with zero spin polarization arranged in two pairs related by a point reflection with respect to the impurity site. It is worthwhile to realize that these plaquettes are completely disconnected in the orbital sector; i.e., there are no orbitally active bonds connecting them. (See Fig. 7 for the pictorial definition of orbitally active bonds.) This lack of active bonds means that quantum effects of a purely orbital nature can appear only at the short range, i.e., inside the plaquettes. However, one can expect that if for some reason the two degenerate spins in a single elementary cell will polarize, then they will also polarize in the same way in all the other cells to favor long-range quantum fluctuations in the spin sector related to the translational invariance of the system.

\section{Phase diagram for periodic $x=1 / 9$ doping}

Finally, we investigate low doping $x=1 / 9$ with a given periodic spatial profile; see Fig. 12. Here, the impurities are separated by the translation vectors $\vec{u}=(0,3)$ and $\vec{v}=(3,0)$. Once again, there is a mismatch between the periodic distribution of impurities and the host's twosublattice AO order, so we again call this doping incommensurate as it also imposes doping at both doublon 


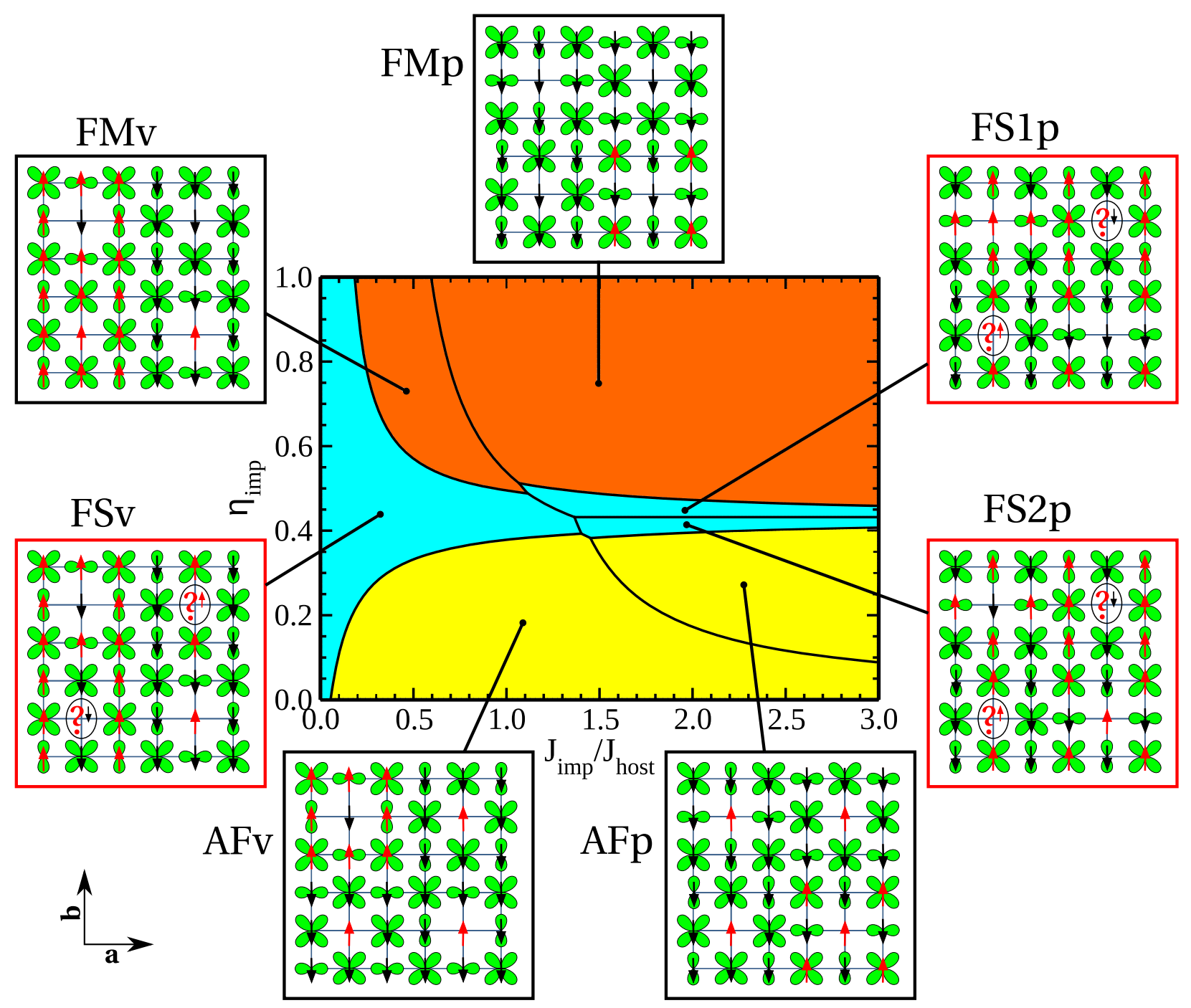

FIG. 12. Ground-state diagram for an $x=1 / 9$ periodic concentration of $3 d$ impurities with schematic views of the ground-state configurations obtained for the unit cell of 36 sites. Spin order (AF, FS, and FM) is highlighted by different colors. The question marks in the FS states (red squares) indicate frustrated impurity spins within the classical approach - the spin is fixed here by quantum fluctuations (small arrows). Doped $3 d$ atoms are at the sites where orbitals are absent.

sublattices. The ground-state diagram presents a gradually increasing tendency toward FM $3 d-4 d$ bonds with increasing $\eta_{\text {imp }}$; see Fig. 12. These polaronic bonds polarize as well the $4 d-4 d$ bonds, and one finds an almost FM order in the $\operatorname{FM} p$ state. Altogether, we have found the same phases as at the higher doping of $x=1 / 5$ (see Fig. 10), i.e., $\mathrm{AF} v$ and $\mathrm{AF} p$ at low values of $\eta_{\text {imp }}$ and $\mathrm{FM} v$ and $\mathrm{FM} p$ in the regime of high $\eta_{\text {imp }}$, separated by the regime of frustrated impurity spins that occur within the phases: $\mathrm{FS} v$, $\mathrm{FS} 1 p$, and FS2 $p$.

The difference between the two AF (FM) states in Fig. 12 is due to the orbital arrangement around the impurity. As for the other doping levels considered so far, $x=1 / 8$ and $x=1 / 5$, we find neutral (inactive) orbitals around $3 d$ impurities in the regime of low $J_{\text {imp }} / J_{\text {host }}$ in the AF $v$ and $\mathrm{FM} v$ phases which lead to spin defects within the 1D FM chains in the $C$-AF spin order. A similar behavior is reported for single impurities in the low- $J_{\text {imp }}$ regime in Sec. III. This behavior changes radically above the orbital transition for both types of local magnetic order, where the orbitals reorient into the active ones. One finds that spin orientations are then the same as those of their neighboring $4 d$ atoms, with some similarities to those found at $x=1 / 5$; see Fig. 10 .

Frustrated impurity spins occur in the crossover regime between the AF and FM local order around impurities following from the local configurations around them, which include two $\uparrow$ spins and two $\downarrow$ spins accompanied by $c$ orbitals at the NN $4 d$ sites. This frustration is easily removed by quantum fluctuations, and we suggest that this happens again in the same way as for $x=1 / 8$ doping, as 
indicated by small arrows in the respective FS phases shown in Fig. 12.

\section{QUANTUM EFFECTS BEYOND THE CLASSICAL APPROACH}

\section{A. Spin-orbital quantum fluctuations}

So far, we have analyzed the ground states of $3 d$ impurities in the $(a, b)$ plane of a $4 d$ system using the classical approach. Here, we show that this classical picture may be used as a guideline and is only quantitatively changed by quantum fluctuations if the spin-orbit coupling is weak. We start the analysis by considering the quantum problem in the absence of spin-orbit coupling (at $\lambda=0$ ). The orbital doublon densities

$$
N_{\gamma} \equiv \sum_{i \in \text { host }}\left\langle n_{i \gamma}\right\rangle
$$

with $\gamma=a, b, c$ and total $S^{z}$ are conserved quantities and thus good quantum numbers for a numerical simulation. To determine the ground-state configurations in the parameter space and the relevant correlation functions, we diagonalize exactly the Hamiltonian matrix (17) for the cluster of $L=8$ sites by means of the Lanczos algorithm. In Fig. 13(a), we report the resulting quantum phase diagram for an eight-site cluster having one impurity and assuming periodic boundary conditions; see Fig. 13(b). The cluster configuration appears to be optimal because it contains a number of sites and connectivities that allow us to analyze separately the interplay between the host-host and the host-impurity interactions and to simulate a physical situation when the interactions within the host dominate over those between the host and the impurity. Such a problem is a quantum analogue of the single unit cell presented in Fig. 9 for $x=1 / 8$ periodic doping.

As a general feature that resembles the classical phase diagram, we observe that there is a prevalent tendency to have AF-like (FM-like) spin correlations between the impurity and the host sites in the region of $\eta_{\text {imp }}$ below (above) the critical point at $\eta_{\mathrm{imp}}^{c} \simeq 0.43$, which separates these two regimes, with intermediate configurations having frustrated magnetic exchange. As we shall discuss below, it is the orbital degree of freedom that turns out to be more

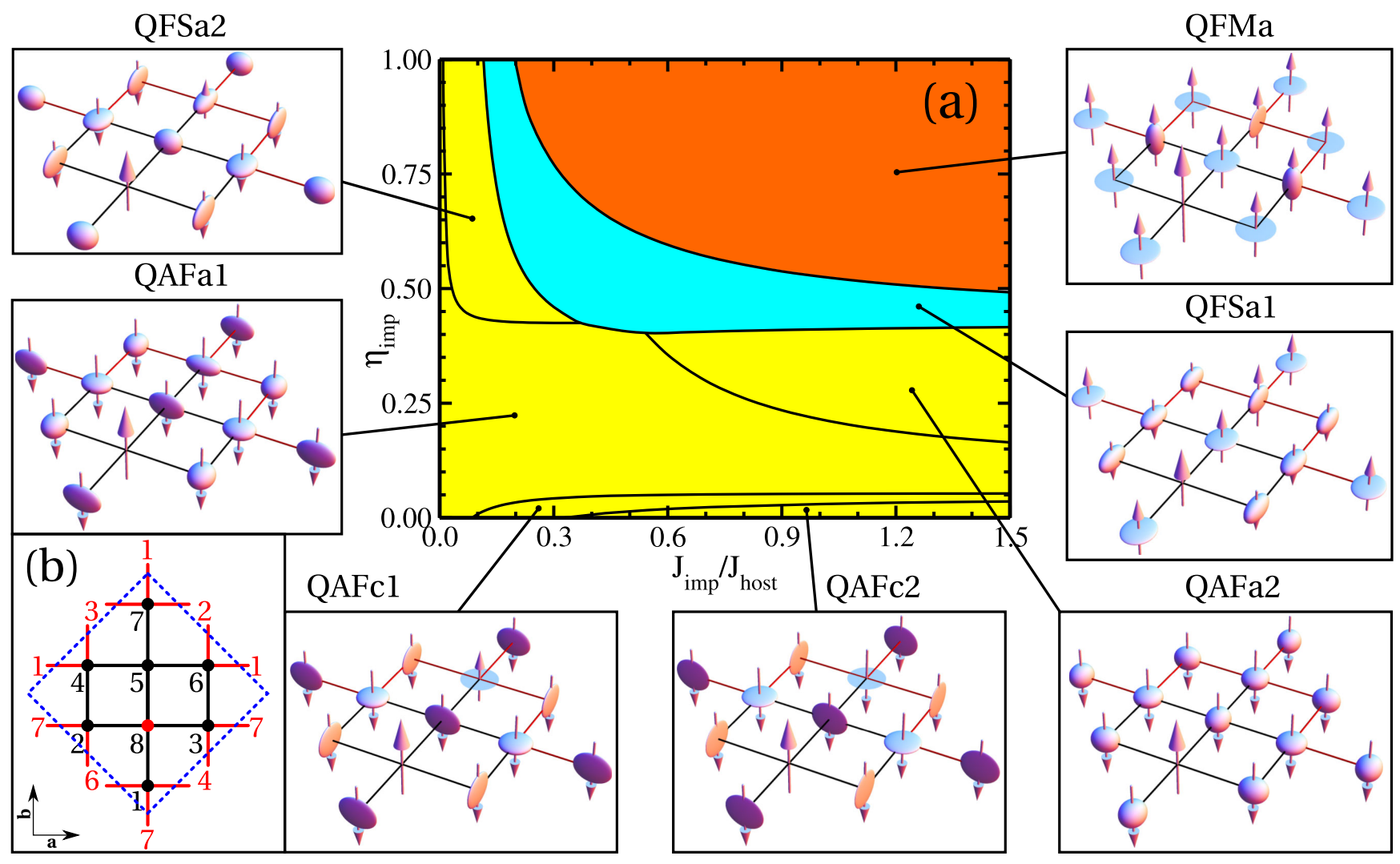

FIG. 13. (a) Phase diagram for the quantum problem at zero spin orbit simulated on the eight-site cluster in the presence of one impurity. Arrows and ellipsoids indicate the spin-orbital state at a given site $i$, while the shapes of ellipsoids reflect the orbital averages: $\left\langle a_{i}^{\dagger} a_{i}\right\rangle,\left\langle b_{i}^{\dagger} b_{i}\right\rangle$, and $\left\langle c_{i}^{\dagger} c_{i}\right\rangle$ (i.e., a circle in the plane perpendicular to the axis $\gamma$ implies 100\% occupation of the orbital $\gamma$ ). (b) The periodic cluster of $L=8$ sites used, with the orbital dilution ( $3 d^{3}$ impurity), at site $i=8$. The dotted lines identify the basic unit cell adopted for the simulation with the same symmetries of the square lattice. 
affected by the quantum effects. Following the notation used for the classical case, we distinguish various quantum AF (QAF) ground states, i.e., QAFc $n(n=1,2)$ and QAFan $(n=1,2)$, as well as a uniform quantum FM (QFM) configuration, i.e., QFM $a$, and a quantum frustrated one labeled as QFSa.

In order to visualize the main spin-orbital patterns contributing to the quantum ground state, it is convenient to adopt a representation with arrows for the spin and ellipsoids for the orbital sector at any given host site. The arrows stand for the on-site spin projection $\left\langle S_{i}^{z}\right\rangle$, with the length being proportional to the amplitude. The length scale for the arrows is the same for all the configurations. Moreover, in order to describe the orbital character of the ground state, we employ a graphical representation that makes use of an ellipsoid whose semiaxis $\{a, b, c\}$ lengths are given by the average amplitude of the squared angular momentum components $\left\{\left(L_{i}^{x}\right)^{2},\left(L_{i}^{y}\right)^{2},\left(L_{i}^{z}\right)^{2}\right\}$ or equivalently by the doublon occupation Eq. (9). For instance, for a completely flat circle (degenerate ellipsoid) lying in the plane perpendicular to the $\gamma$ axis, only the corresponding $\gamma$ orbital is occupied. On the other hand, if the ellipsoid develops in all three directions $\{a, b, c\}$, it implies that more than one orbital is occupied and the distribution can be anisotropic in general. If all the orbitals contribute equally, one finds an isotropic spherical ellipsoid.

Because of the symmetry of the Hamiltonian, the phases shown in the phase diagram of Fig. 13(a) can be characterized by the quantum numbers for the $z$ th spin projection $S^{z}$ and the doublon orbital occupation $N_{\alpha}(25),\left(S^{z}, N_{a}, N_{b}, N_{c}\right): \operatorname{QAF} c 1(-3.5,2,2,3)$, $\mathrm{QFS} a 2(-1.5,3,1,3), \mathrm{QAF} a 1(-5.5,1,3,3), \mathrm{QAF} a 2$ and $\mathrm{QAF} c 2(-5.5,2,2,3), \mathrm{QFS} a 1(-0.5,3,0,4)$, and QFM $a$ $(-8.5,2,1,4)$. Despite the irregular shape of the cluster [Fig. 13(b)], there is also symmetry between the $a$ and $b$ directions. For this reason, the phases with $N_{a} \neq N_{b}$ can be equivalently described either by the set $\left(S^{z}, N_{a}, N_{b}, N_{c}\right)$ or $\left(S^{z}, N_{b}, N_{a}, N_{c}\right)$.

The outcome of the quantum analysis indicates that the spin patterns are quite robust, as the spin configurations of the phases $\mathrm{QAF} a, \mathrm{QAF} c, \mathrm{QFS} a$, and $\mathrm{QFM} a$ are the analogues of the classical ones. The effects of quantum fluctuations are more evident in the orbital sector where mixed orbital patterns occur if compared to the classical case. In particular, orbital inactive states around the impurity are softened by quantum fluctuations and on some bonds we find an orbital configuration with a superposition of active and inactive states. The unique $\mathrm{AF}$ states where the classical inactive scenario is recovered correspond to the $\mathrm{QAF} c 1$ and $\mathrm{QAF} c 2$ ones in the regime of small $\eta_{\text {imp. }}$. A small hybridization of active and inactive orbitals along both the AF and FM bonds is also observed around the impurity for the QFS $a$ phases as one can note by the shape of the ellipsoid at host sites. Moreover, in the range of large $\eta_{\text {imp }}$ where the FM state is stabilized, the orbital pattern around the impurity is again like in the classical case.

A significant orbital rearrangement is also obtained within the host. We generally obtain an orbital pattern that is slightly modified from the pure $\mathrm{AO}$ configuration assumed in the classical case. The effect is dramatically different in the regime of strong impurity-host coupling (i.e., for large $J_{\text {imp }}$ ) with $\mathrm{AF}$ exchange (QAFa2) with the formation of an orbital liquid around the impurity and within the host, with doublon occupation represented by an almost isotropic shaped ellipsoid. Interestingly, though with a different orbital arrangement, the QFS 1 and the QFS $a 2$ states are the only ones where the $C$-AF order of the host is recovered. For all the other phases shown in the diagram of Fig. 13, the coupling between the host and the impurity is generally leading to a uniform spin polarization with FM or AF coupling between the host and the impurity depending on the strength of the host-impurity coupling. Altogether, we conclude that the classical spin patterns are only quantitatively modified and are robust with respect to quantum fluctuations.

\section{B. Finite spin-orbit coupling}

In this section, we analyze the quantum effects in the spin and orbital order around the impurity in the presence of the spin-orbit coupling at the host $d^{4}$ sites. For the $t_{2 g}^{4}$ configuration, the strong spin-orbit regime has been considered recently by performing an expansion around the atomic limit where the angular $\vec{L}_{i}$ and spin $\vec{S}_{i}$ momenta form a spin-orbit singlet for the amplitude of the total angular momentum $\vec{J}_{i}=\vec{L}_{i}+\vec{S}_{i}$ (i.e., $J=0$ ) [67]. The instability toward an $\mathrm{AF}$ state starting from the $J=0$ liquid has been obtained within the spin-wave theory [68] for the low-energy excitations emerging from the spin-orbital exchange.

In the analysis presented here, we proceed from the limit of zero spin orbit to investigate how the spin and orbital order are gradually suppressed when approaching the $J=0$ spin-orbit-singlet state. This issue is addressed by solving the full quantum Hamiltonian (17) exactly on a cluster of $L=8$ sites including the spin-orbital exchange for the host and that one derived for the hostimpurity coupling (17) as well as the spin-orbit term

$$
\mathcal{H}_{\mathrm{SO}}=\lambda \sum_{i \in \text { host }} \vec{L}_{i} \cdot \vec{S}_{i}
$$

where the sum includes the ions of the $4 d$ host and we use the spin $S=1$ and the angular momentum $L=1$, as in the ionic $4 d^{4}$ configurations. Here, $\lambda$ is the spin-orbitcoupling constant at $4 d$ host ions, and the components of the orbital momentum $\vec{L}_{i} \equiv\left\{L_{i}^{x}, L_{i}^{y}, L_{i}^{z}\right\}$ are defined as follows: 


$$
\begin{aligned}
& L_{i}^{x}=i \sum_{\sigma}\left(d_{i, x y \sigma}^{\dagger} d_{i, x z \sigma}-d_{i, x z \sigma}^{\dagger} d_{i, x y \sigma}\right), \\
& L_{i}^{y}=i \sum_{\sigma}\left(d_{i, x y \sigma}^{\dagger} d_{i, y z \sigma}-d_{i, y z \sigma}^{\dagger} d_{i, x y \sigma}\right), \\
& L_{i}^{z}=i \sum_{\sigma}\left(d_{i, x z \sigma}^{\dagger} d_{i, y z \sigma}-d_{i, y z \sigma}^{\dagger} d_{i, x z \sigma}\right) .
\end{aligned}
$$

To determine the ground state and the relevant correlation functions, we use again the Lanczos algorithm for the cluster of $L=8$ sites. Such an approach allows us to study the competition between the spin-orbital exchange and the spin-orbit coupling on equal footing without any simplifying approximation. Moreover, the cluster calculation permits us to include the impurity in the host and deal with the numerous degrees of freedom without making approximations that would constrain the interplay of the impurity-host versus host-host interactions.

Finite spin-orbit coupling significantly modifies the symmetry properties of the problem. Instead of the $\mathrm{SU}(2)$ spin invariance, one has to deal with the rotational invariance related to the total angular momentum per site $\vec{J}_{i}=\vec{L}_{i}+\vec{S}_{i}$. Although the $\vec{L}_{i} \cdot \vec{S}_{i}$ term in Eq. (26) commutes with both total $\vec{J}^{2}$ and $J^{z}$, the full Hamiltonian for the host with impurities Eq. (17) has a reduced symmetry because the spin sector is now linearly coupled to the orbital, which has only the cubic symmetry. Thus, the remaining symmetry is a cyclic permutation of the $\{x, y, z\}$ axes.

Moreover, $J^{z}$ is not a conserved quantity due to the orbital anisotropy of the spin-orbital exchange in the host and the orbital character of the impurity-host coupling. There, one has a $\mathbb{Z}_{2}$ symmetry associated with the parity operator $(-1)^{J_{z}}$. Hence, the ground state can be classified as even or odd with respect to the value of $J^{z}$. This symmetry aspect can introduce a constraint on the character of the ground state and on the impurity-host coupling since the $J^{z}$ value for the impurity is only due to the spin projection while in the host it is due to the combination of the orbital and spin projection. A direct consequence is that the parity constraint together with the unbalance between the spin at the host and the impurity sites leads to a nonvanishing total projection of the spin and angular momentum with respect to a symmetry axis, e.g., the zth axis. It is worth noting that a fixed parity for the impurity spin means that it prefers to point in one direction rather than the other one, which is not the case for the host's spin and angular momentum. Thus, the presence on the impurity for a fixed $\mathcal{P}$ will give a nonzero polarization along the quantization axis for every site of the system. Such a property holds for any single impurity with a half-integer spin.

Another important consequence of the spin-orbit coupling is that it introduces local quantum fluctuations in the orbital sector even at the sites close to the impurity where the orbital pattern is disturbed. The spin-orbit term makes the on-site problem around the impurity effectively analogous to the Ising model in a transverse field for the orbital sector, with nontrivial spin-orbital entanglement [34] extending over the impurity neighborhood.

In Figs. 14 and 15, we report the schematic evolution of the ground-state configurations for the cluster of $L=8$ sites, with one-impurity and periodic boundary conditions as a function of increasing spin-orbit coupling. These patterns have been determined by taking into account the sign and the amplitude of the relevant spatial dependent spin and orbital correlation functions. The arrows associated with the spin degree of freedom can lie in the $x y$ plane or out of plane (along $z$, chosen to be parallel to the $c$ axis) to indicate the anisotropic spin pattern. The out-of-plane arrow length is given by the on-site expectation value of $\left\langle S_{i}^{z}\right\rangle$ while the in-plane arrow length is obtained by computing the square root of the second moment, i.e., $\sqrt{\left\langle\left(S_{i}^{x}\right)^{2}\right\rangle}$ and $\sqrt{\left\langle\left(S_{i}^{y}\right)^{2}\right\rangle}$ of the $x$ and $y$ spin components corresponding to the arrows along $a$ and $b$, respectively.

Moreover, the in-plane arrow orientation for a given direction is determined by the sign of the corresponding spin-spin correlation function assuming as a reference the orientation of the impurity spin. The ellipsoid is constructed in the same way as for the zero spin-orbit case above, with the addition of a color map that indicates the strength of the average $\vec{L}_{i} \cdot \vec{S}_{i}$ (i.e., red, yellow, green, blue, and violet correspond with a growing amplitude of the local spin-orbit correlation function). The scale for the spin-orbit amplitude is set to be in the interval $0<\lambda<J_{\text {host }}$. The selected values for the ground-state evolution are given by the relation (with $m=1,2, \ldots, 10$ )

$$
\lambda_{m}=\left[0.04+0.96 \frac{(m-1)}{9}\right] J_{\text {host }} .
$$

The scale is set such that $\lambda_{1}=0.04 J_{\text {host }}$ and $\lambda_{10}=J_{\text {host }}$. This range of values allows us to explore the relevant physical regimes when moving from $3 d$ to $4 d$ and $5 d$ materials with corresponding $\lambda$ being much smaller that $J_{\text {host }}: \lambda \sim J_{\text {host }} / 2$ and $\lambda>J_{\text {host }}$, respectively. For the performed analysis, the selected values of $\lambda$ (28) are also representative of the most interesting regimes of the ground state as induced by the spin-orbit coupling.

Let us start with the quantum $\mathrm{AF}$ phases $\mathrm{QAF} c 1$, $\mathrm{QAF} c 2$, QFSa1, QFSa2, QAFa1, and QAFa2. As one can observe, the switching on of the spin-orbit coupling (i.e., $\lambda_{1}$ in Fig. 14) leads to anisotropic spin patterns with unequal moments for the in-plane and out-of-plane components. From weak to strong spin-orbit coupling, the character of the spin correlations keeps being AF between the impurity and the neighboring host sites in all the spin directions. The main change for the spin sector occurs for the planar components. For weak spin-orbit coupling, the in-plane spin pattern is generally $\mathrm{AF}$ for the whole system 


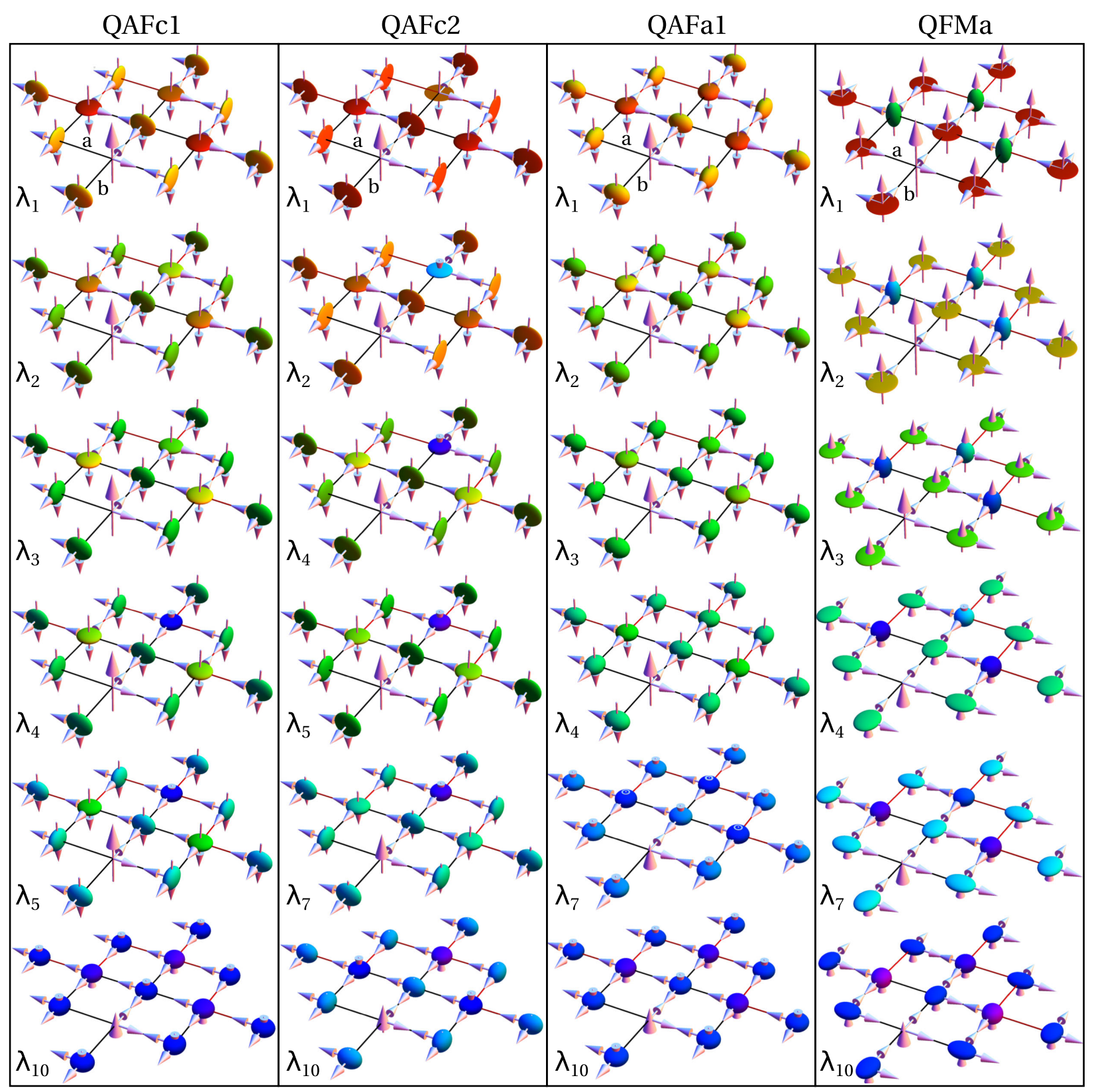

FIG. 14. Evolution of the ground-state configurations for the AF phases for selected increasing values of spin-orbit coupling $\lambda_{m}$; see Eq. (28). Arrows and ellipsoids indicate the spin-orbital state at a given site $i$. The color map indicates the strength of the average spin orbit $\left\langle\vec{L}_{i} \cdot \vec{S}_{i}\right\rangle$; i.e., red, yellow, green, blue, and violet correspond to the growing amplitude of the above correlation function. Small arrows at $\lambda_{5}$ and $\lambda_{10}$ indicate quenched magnetization at the impurity by large spin-orbit coupling at the neighboring host sites.

in all the spatial directions (i.e., $G$-AF order). Further increase of the spin orbit does not modify qualitatively the character of the spin pattern for the out-of-plane components as long as we do not go to maximal values of $\lambda \sim J_{\text {host }}$ where local $\left\langle S_{i}^{z}\right\rangle$ moments are strongly suppressed. In this limit, the dominant tendency of the system is toward formation of the spin-orbital singlets and the spin patterns shown in Fig. 14 are the effect of the virtual singlet-triplet excitations [68].

Concerning the orbital sector, only for weak spin-orbit coupling around the impurity can one still observe a reminiscence of inactive orbitals as related to the orbital vacancy role at the impurity site in the AF phase. Such an orbital configuration is quickly modified by increasing the spin-orbit interaction, and it evolves into a uniform pattern with almost degenerate orbital occupations in all the directions and with preferential superpositions of $c$ and $(a, b)$ states associated with dominating $L^{x}$ and $L^{y}$ orbital angular components (flattened ellipsoids along the $c$ direction). An exception is the $\mathrm{QAF} c 2$ phase with the orbital inactive polaron that is stable up to large spin-orbit coupling of the order of $J_{\text {host }}$. 


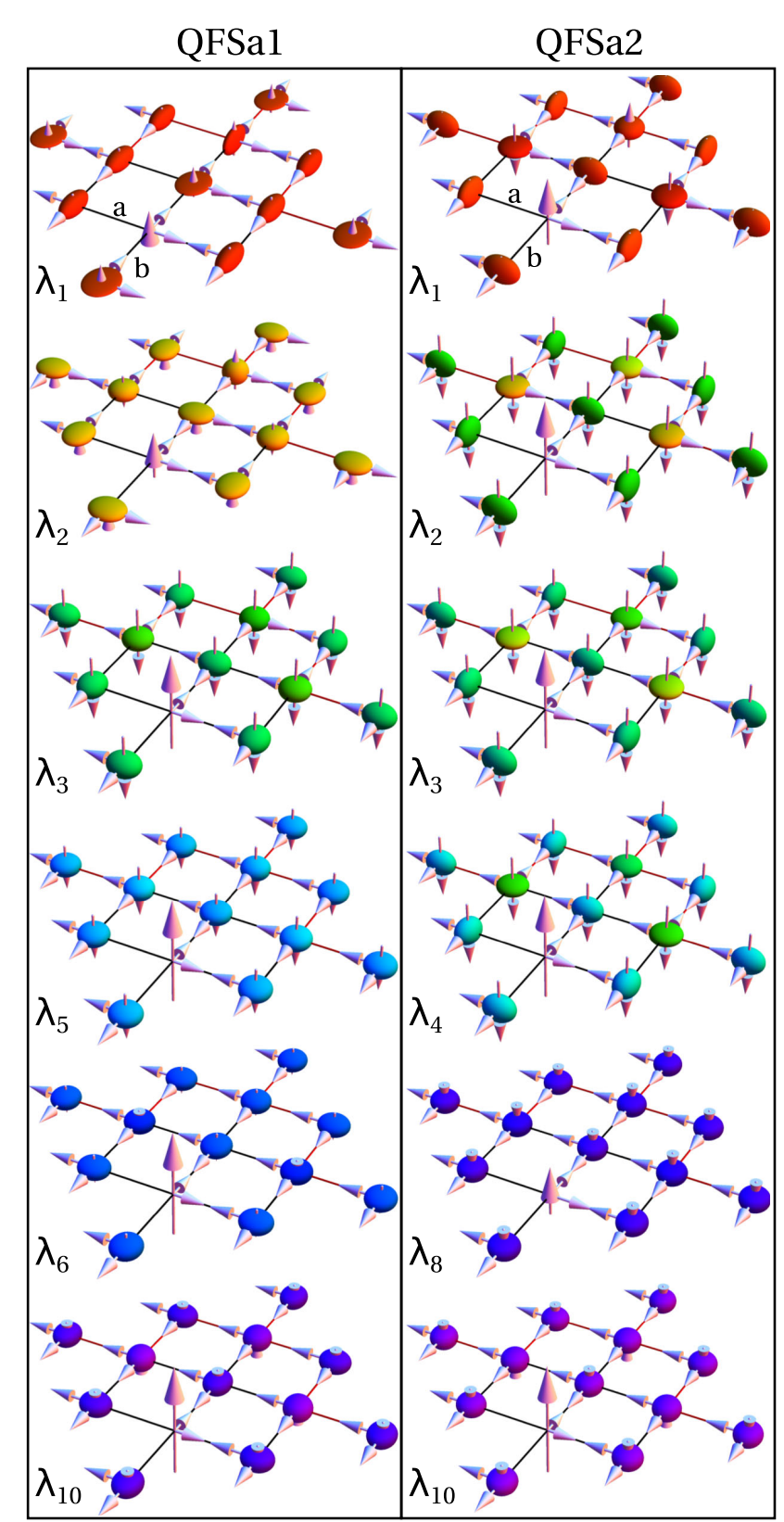

FIG. 15. Evolution of the ground-state configurations for the QFS $a 1$ and QFS $a 2$ phases for selected increasing values of spinorbit coupling $\lambda_{m}$; see Eq. (28). Arrows and ellipsoids indicate the spin-orbital state at a given site $i$. The color map indicates the strength of the average spin orbit $\left\langle\vec{L}_{i} \cdot \vec{S}_{i}\right\rangle$; i.e., red, yellow, green, blue, and violet correspond to the growing amplitude of the above local correlation function.

When considering the quantum FM configurations QFM $a 1$ in Fig. 14, we observe similar trends in the evolution of the spin correlation functions as obtained for the AF states. Indeed, the QFM $a$ exhibits a tendency to form FM chains with AF coupling for the in-plane components at weak spin orbit that evolve into more dominant AF correlations in all the spatial directions within the host. Interestingly, the spin exchange between the impurity and the neighboring host sites shows a changeover from AF to FM for the range of intermediate-to-strong spinorbit amplitudes.

A peculiar response to the spin-orbit coupling is obtained for the QFSa1 phase (see Fig. 15), which showed a frustrated spin pattern around the impurity already in the classical regime, with FM and AF bonds. It is remarkable that due to the close proximity with uniform FM and the $\mathrm{AF}$ states, the spin-orbit interaction can lead to a dramatic rearrangement of the spin and orbital correlations for such a configuration. At weak spin-orbit coupling (i.e., $\lambda \simeq \lambda_{1}$ ), the spin pattern is $C$-AF and the increased coupling $\left(\lambda \simeq \lambda_{2}\right)$ keeps the $C$-AF order only for the in-plane components with the exception of the impurity site. It also modulates the spin-moment distribution around the impurity along the $z$ direction. Further increase of $\lambda$ leads to complete spin polarization along the $z$ direction in the host, with antiparallel orientation with respect to the impurity spin. This pattern is guided by the proximity to the FM phase. The in-plane components develop a mixed FM-AF pattern with a strong $x y$ anisotropy most probably related to the different bond exchange between the impurity and the host.

When approaching the regime of a spin-orbit coupling that is comparable to $J_{\text {host }}$, the out-of-plane spin components dominate and the only out-of-plane spin polarization is observed at the impurity site. Such a behavior is unique and occurs only in the QFS $a$ phases. The cooperation between the strong spin-orbit coupling and the frustrated host-impurity spin-orbital exchange leads to an effective decoupling in the spin sector at the impurity with a resulting maximal polarization. On the other hand, as for the AF states, the most favorable configuration for a strong spin orbit has AF in-plane spin correlations. The orbital pattern for the QFS $a$ states evolves similarly to the AF cases with a suppression of the active-inactive interplay around the impurity and the setting of a uniformlike orbital configuration with unquenched angular momentum on site and predominant in-plane components. The response of the FM state is different in this respect, as the orbital active states around the impurity are hardly affected by the spin orbit while the host sites far from the impurity of the local spin-orbit coupling are more pronounced.

Finally, to understand the peculiar evolution of the spin configuration, it is useful to consider the lowest-order terms in the spin-orbital exchange that directly couple the orbital angular momentum with the spin. Taking into account the expression of the spin-orbital exchange in the host (26) and the expression of $\vec{L}_{i}$, one can show the low-energy terms on a bond that get more relevant in the Hamiltonian when the spin-orbit coupling makes a nonvanishing local angular momentum. As a result, the corresponding expressions are

$$
\begin{aligned}
H_{\text {host }}^{a(b)}(i, j) \approx & J_{\text {host }}\left\{a_{1} \vec{S}_{i} \cdot \vec{S}_{j}+b_{1} S_{i}^{z} S_{j}^{z} L_{i}^{y(x)} L_{j}^{y(x)}\right\} \\
& +\lambda\left\{\vec{L}_{i} \cdot \vec{S}_{i}+\vec{L}_{j} \cdot \vec{S}_{j}\right\}
\end{aligned}
$$


with positive coefficients $a_{1}$ and $b_{1}$ that depend on $r_{1}$ and $r_{2}$ (21). A definite sign for the spin exchange in the limit of vanishing spin-orbit coupling is given by the terms that go beyond Eq. (29). Then, if the ground state has isotropic FM correlations (e.g., QFM $a$ ) at $\lambda=0$, the term $S_{i}^{z} S_{j}^{z} L_{i}^{y(x)} L_{j}^{y(x)}$ would tend to favor AF-like configurations for the in-plane orbital angular components when the spin-orbit interaction is switched on. This opposite tendency between the $z$ and $\{x, y\}$ components is counteracted by the local spin-orbit coupling that prevents us from having coexisting FM and AF spin-orbital correlations. Such patterns would not allow us to optimize the $\vec{L}_{i} \cdot \vec{S}_{i}$ amplitudes. One way out is to reduce the $z$ th spin projection and to get planar AF correlations in the spin and in the host. A similar reasoning applies to the AF states where the negative sign of the $S_{i}^{z} S_{j}^{z}$ correlations favors ferro-orbital alignment of the angular momentum components. As for the previous case, the opposite trend of in- and out-of-plane spin-orbital components is suppressed by the spin-orbit coupling and the in-plane ferro-orbital correlations for the $\left\{L^{x}, L^{y}\right\}$ components lead to FM patterns for the in-plane spin part as well.

In summary, by close inspection of Figs. 14 and 15, one finds an interesting evolution of the spin patterns in the quantum phases.

(i) For the QAF states (Fig. 14), a spin canting develops at the host sites (i.e., the relative angle is between 0 and $\pi$ ) while the spins on impurity-host bonds are always AF. The canting in the host evolves, sometime in an inhomogeneous way, to become reduced in the strong spin-orbit-coupling regime where ferrolike correlations tend to dominate. In this respect, when the impurity is coupled antiferromagnetically to the host, it does not follow the tendency to form spin canting.

(ii) In the QFM states (Fig. 15), at weak spin orbit, one observes spin canting in the host and for the hostimpurity coupling that persists only in the host, whereas the spin-orbit interaction is increasing.

\section{Spin-orbit coupling versus Hund's exchange}

To probe the phase diagram of the system in the presence of the spin-orbit coupling $(\lambda>0)$, we solve the same cluster of $L=8$ sites as before along three different cuts in the phase diagram of Fig. 13(a) for three values of $\lambda$, i.e., small $\lambda=0.1 J_{\text {host }}$, intermediate $\lambda=0.5 J_{\text {host }}$, and large $\lambda=J_{\text {host }}$. Each cut contains ten points, and the cuts are parameterized as follows: (i) $J_{\text {imp }}=0.7 J_{\text {host }}$ and $0 \leq \eta_{\text {imp }} \leq 0.7$, (ii) $J_{\text {imp }}=1.3 J_{\text {host }}$ and $0 \leq \eta_{\text {imp }} \leq 0.7$, and (iii) $\eta_{\text {imp }}=\eta_{\text {imp }}^{c} \simeq 0.43$ and $0 \leq J_{\text {imp }} \leq 1.5 J_{\text {host }}$. In Fig. 16(a), we show the representative spin-orbital configurations obtained for $\lambda=0.5 J_{\text {host }}$ along the first cut shown in Fig. 16(b). Values of $\eta_{\text {imp }}$ are chosen as

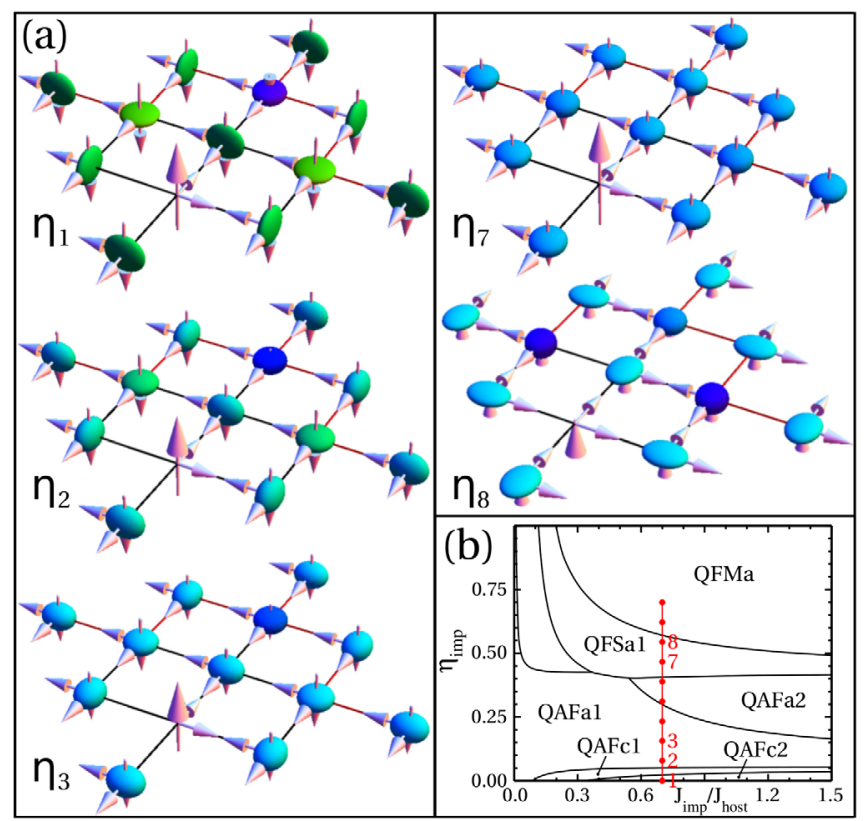

FIG. 16. (a) Evolution of the ground-state configurations as for increasing $\eta_{\mathrm{imp}}$ and for a fixed value of spin-orbit coupling $\lambda=0.5 J_{\text {host }}$ along a cut in the phase diagram shown in (b), i.e., for $J_{\text {imp }}=0.7 J_{\text {host }}$ and $0 \leq \eta_{\text {imp }} \leq 0.7$. Arrows and ellipsoids indicate the spin-orbital state at a given site $i$. The color map indicates the strength of the average spin orbit $\left\langle\vec{L}_{i} \cdot \vec{S}_{i}\right\rangle$; i.e., red, yellow, green, blue, and violet correspond to the growing amplitude of the above correlation function.

$$
\eta_{\text {imp }}=\eta_{m} \equiv 0.7 \frac{(m-1)}{9}
$$

with $m=1, \ldots, 10$, but not all the points are shown in Fig. 16(a) - only the ones for which the spin-orbital configuration changes substantially.

The cut starts in the QAFc2 phase, according to the phase diagram of Fig. 16(b), and indeed we find a similar configuration to the one shown in Fig. 14 for the QAFc2 phase at $\lambda=\lambda_{5}$. Moving up in the phase diagram from $\eta_{1}$ to $\eta_{2}$, we see that the configuration evolves smoothly to the one that we have found in the QAF $a 1$ phase at $\lambda=\lambda_{5}$ (not shown in Fig. 14). The evolution of spins is such that the out-of-plane moments are suppressed while in-plane ones are slightly enhanced. The orbitals become more spherical, and the local spin-orbit average $\left\langle\vec{L}_{i} \cdot \vec{S}_{i}\right\rangle$ becomes larger and more uniform; however, for the apical site $i=7$ in the cluster [Fig. 13(b)], the trend is the opposite-initially, the large value of the spin-orbit coupling drops toward the uniform value. We skip the points between $\eta_{3}$ and $\eta_{7}$, as the evolution is smooth and the trend is clear; however, the impurity out-of-plane moment begins to grow above $\eta_{5}$, indicating proximity to the $\mathrm{QFS} a 1$ phase. For this phase at intermediate and high $\lambda$, the impurity moment is much larger than all the others (see Fig. 15). 
For $\eta_{\text {imp }}=\eta_{7}$, the orbital pattern clearly shows that we are in the QFS $a 1$ phase at $\lambda=\lambda_{5}$, which agrees with the position of the $\eta_{7}$ point in the phase diagram; see Fig. 16(b). On the other hand, moving to the next $\eta_{\text {imp }}$ point upward along the cut [Eq. (30)], we already observe a configuration that is very typical for the QFS $a 1$ phase at intermediate $\lambda$. (Here, $\lambda=\lambda_{7}$, as shown in Fig. 14, but also $\lambda_{6}$, which is not shown.) This indicates that the QFSa1 phase can still be distinguished at $\lambda=0.5 J_{\text {host }}$, and its position in the phase diagram is similar to the $\lambda=0$ case, i.e., as an intermediate phase between the QAFa1(2) and the QFM $a$ one.

Finally, we have also found that the two other cuts that were not shown here, i.e., for $J_{\text {imp }}=1.3 J_{\text {host }}$ and increasing $\eta_{\text {imp }}$ and for $\eta_{\text {imp }}=\eta_{\text {imp }}^{c} \simeq 0.43$ and increasing $J_{\text {imp }}$, confirm that the overall character of the phase diagram of Fig. 13(a) is preserved at this value of spin-orbit coupling; however, first, the transitions between the phases are smooth, and second, the subtle differences between the two versions of $\mathrm{QFS} a, \mathrm{QAF} a$, and $\mathrm{QAF} c$ phases are no longer present. The situation is similar for the smaller value of $\lambda$, i.e., $\lambda=0.1 J_{\text {host }}$, but already for $\lambda=J_{\text {host }}$ the out-ofplane moments are so strongly suppressed (except for the impurity moment in the QFS $a 1$ phase) and the orbital polarization is so weak (i.e., almost spherical ellipsoids) that typically the only distinction between the phases can be made by looking at the in-plane spin correlations and the average spin orbit $\left\langle\vec{L}_{i} \cdot \vec{S}_{i}\right\rangle$. In this limit, we conclude that the phase diagram is (partially) melted by large spin-orbit coupling, but for lower values of $\lambda$, it is still valid.

\section{SUMMARY AND CONCLUSIONS}

We have derived the spin-orbital superexchange model for $3 d^{3}$ impurities replacing $4 d^{4}$ (or $3 d^{2}$ ) ions in the $4 d(3 d)$ host in the regime of the Mott-insulating phase. Although the impurity has no orbital degree of freedom, we have shown that it contributes to the spin-orbital physics and strongly influences the orbital order. In fact, it tends to project out the inactive orbitals at the impurity-host bonds to maximize the energy gain from virtual charge fluctuations. In this case, the interaction along the superexchange bond can be either antiferromagnetic or ferromagnetic, depending on the ratio of Hund's exchange coupling at impurity $\left(J_{1}^{H}\right)$ and host $\left(J_{2}^{H}\right)$ ions and on the mismatch $\Delta$ between the $3 d$ and $4 d$ atomic energies, modified by the difference in Hubbard $U$ 's and Hund's exchange $J^{H}$ 's at both atoms. This ratio, denoted by $\eta_{\text {imp }}(14)$, replaces here the conventional parameter $\eta=J_{H} / U$ often found in the spin-orbital superexchange models of undoped compounds (e.g., in the Kugel-Khomskii model for $\mathrm{KCuF}_{3}$ [14]), where it quantifies the proximity to ferromagnetism. On the other hand, if the overall coupling between the host and impurity is weak in the sense of the total superexchange $J_{\text {imp }}$ with respect to the host value $J_{\text {host }}$, the orbitals next to the impurity may be forced to stay inactive, which modifies the magnetic properties - in such cases, the impurity-host bond is always antiferromagnetic.

As we have seen in the case of a single impurity, the above two mechanisms can have a nontrivial effect on the host, especially if the host itself is characterized by frustrated interactions, as it happens in the parameter regime where the $C$-AF phase is stable. For this reason, we have mostly focused on the latter phase of the host and we have presented the phase diagrams of a single-impurity configuration in the case when the impurity is doped on the sublattice where the orbitals form a checkerboard pattern with alternating $c$ and $a$ orbitals occupied by doublons. The diagram for the $c$-sublattice doping shows that in some sense, the impurity is never weak because even for a very small value of $J_{\text {imp }} / J_{\text {host }}$, it can release the host's frustration around the impurity site acting as an orbital vacancy. On the other hand, for the $a$-sublattice doping when the impurityhost coupling is weak, i.e., either $J_{\text {imp }} / J_{\text {host }}$ is weak or $\eta_{\text {imp }}$ is close to $\eta_{\text {imp }}^{c}$, we have identified an interesting quantum mechanism releasing frustration of the impurity spin (that cannot be avoided in the purely classical approach). It turns out that in such situations, the orbital flips in the host make the impurity spin polarize in such a way that the $C$-AF order of the host is completely restored.

The cases of the periodic doping studied in this paper show that the host's order can be completely altered already for rather low doping of $x=1 / 8$, even if the $J_{\text {imp }} / J_{\text {host }}$ is small. In this case, we can stabilize a ferrimagnetic type of phase with a four-site unit cell having magnetization $\left\langle S_{i}^{z}\right\rangle=3 / 2$, reduced further by quantum fluctuations. We have established that the only parameter range where the host's order remains unchanged is when $\eta_{\text {imp }}$ is close to $\eta_{\text {imp }}^{c}$ and $J_{\text {imp }} / J_{\text {host }} \gtrsim 1$. The latter value is very surprising, as it means that the impurity-host coupling must be large enough to keep the host's order unchanged-which is another manifestation of the orbital vacancy mechanism that we have already observed for a single impurity. Also, in this case, the impurity spins are fixed with the help of orbital flips in the host that lift the degeneracy that arises in the classical approach. We would like to point out that the quantum mechanism that lifts the ground-state degeneracy mentioned above and the role of quantum fluctuations are of particular interest for the periodically doped checkerboard systems with $x=1 / 2$ doping, which is a challenging problem for future research.

From the point of view of generic, i.e., nonperiodic, doping, the most representative cases are those of a doping that is incommensurate with the two-sublattice spin-orbital pattern. To uncover the generic rules in such cases, we have studied periodic $x=1 / 5$ and $x=1 / 9$ doping. One finds that when the period of the impurity positions does not match the period of 2 for both the spin and orbital orders of the host, interesting novel types of order emerge. In such cases, the elementary cell must be doubled in both lattice directions, which clearly gives a chance of realizing more 
phases than in the case of commensurate doping. Our results show that indeed, the number of phases increases from 4 to 7 and the host's order is altered in each of them. Quite surprisingly, the overall character of the phase diagram remains unchanged with respect to the one for $x=1 / 8$ doping, and, if we ignore the differences in configuration, it seems that only some of the phases get divided into two versions differing either by the spin bond's polarizations around impurities (phases around $\eta_{\text {imp }}^{c}$ ) or by the character of the orbitals around the impurities (phases with inactive orbitals in the limit of small enough product $\eta_{\text {imp }} J_{\text {imp }}$ versus phases with active orbitals in the opposite limit). Orbital polarization in this latter region resembles orbital polarons in doped manganites $[42,43]$-also, here, such states are stabilized by the double exchange [46].

A closer inspection of underlying phases reveals, however, a very interesting degeneracy of the impurity spins at $x=1 / 5$ that arises again from the classical approach, but this time, it cannot be released by short-range orbital flips. The degeneracy persists because the host's order is already so strongly altered that it is no longer anisotropic (as is the case of the $C$-AF phase) and there is no way to restore the orbital anisotropy around the impurities that could lead to spin-bond imbalance and polarize the spin. In the case of lower $x=1 / 9$ doping, such an effect is absent and the impurity spins are always polarized, as happens for $x=1 / 8$. It shows that such robust degeneracy is rather a peculiarity of the $x=1 / 5$ periodic doping.

Indeed, one can easily notice that for $x=1 / 5$, every atom of the host is a nearest neighbor of some impurity. In contrast, for $x=1 / 8$, we can find three host's atoms per unit cell that do not neighbor any impurity, and for $x=1 / 9$, there are 16 of them. For this reason, the impurity effects are amplified for $x=1 / 5$, which is not unexpected, although one may find it somewhat surprising that the ground-state diagrams for the lowest and highest doping considered here are very similar. Such similarity suggests that the cooperative effects of multiple impurities are indeed not very strong in the low-doping regime, so the diagram obtained for $x=1 / 9$ can be regarded as generic for the dilute doping regime with uniform spatial profile.

For the representative case of $x=1 / 8$ doping, we have presented the consequences of quantum effects beyond the classical approach. Spin fluctuations are rather weak for the considered case of large $S=1$ and $S=3 / 2$ spins, and we have shown that orbital fluctuations on superexchange bonds are more important. They are strongest in the regime of antiferromagnetic impurity-host coupling (which suggests the importance of entangled states [34]) and enhance the tendency toward frustrated impurity-spin configurations but do not destroy other generic trends observed when the parameters $\eta_{\text {imp }}$ and $J_{\text {imp }} / J_{\text {host }}$ increase.

Increasing spin-orbit coupling leads to qualitative changes in the spin-orbital order. When Hund's exchange is small at the impurity sites, the antiferromagnetic bonds around it have reduced values of the spin-orbit-coupling term, but the magnetic moments reorient and survive in the $(a, b)$ planes, with some similarity to the phenomena occurring in the perovskite vanadates [57]. As a consequence, the magnetic moments at $3 d$ impurities are quenched and orbital occupancies at the host sites are almost uniform. In contrast, frustration of impurity spins is removed and the impurity magnetization along the $c$ axis survives for large spin-orbit coupling.

We would like to emphasize that the orbital dilution considered here influences directly the orbital degrees of freedom in the host around the impurities. The synthesis of hybrid compounds having both $3 d$ and $4 d$ transition-metal ions will likely open a novel route for unconventional effects in complex materials. There are several reasons for expecting new scenarios in mixed $3 d-4 d$ spin-orbitallattice materials, and we pointed out only some of them. On the experimental side, the changes of local order could be captured using inelastic neutron scattering or resonant inelastic x-ray scattering (RIXS). In fact, using RIXS can also bring an additional advantage: RIXS, besides being a perfect probe of both spin and orbital excitations, can also (indirectly) detect the nature of the orbital ground state (supposedly also including the nature of impurities in the crystal) [82]. Unfortunately, there are no such experiments yet, but we believe that they will be available soon.

Short-range order around impurities could be investigated by the excitation spectra at the resonant edges of the substituting atoms. Taking them both at finite energy and momentum can provide insights into the nature of the shortrange order around the impurity and then unveil information of the order within the host as well. Even if there are no elastic superlattice extra peaks, one can expect that the spinorbital correlations will emerge in the integrated RIXS spectra providing information of the impurity-host coupling and of the short-range order around the impurity. Even more interesting is the case where the substituting atom forms a periodic array with small deviation from the perfect superlattice when one expects the emergence of extra elastic peaks that will clearly indicate the spin-orbital reconstruction. In our case, an active orbital diluted site cannot participate coherently in the host spin-orbital order but rather may restructure the host ordering [83]. At dilute impurity concentration, we may expect broad peaks emerging at finite momenta in the Brillouin zone, indicating the formation of coherent islands with short-range order around impurities.

We also note that local susceptibility can be suitably measured by making use of resonant spectroscopies (e.g., nuclear magnetic resonance, electron spin resonance, nuclear quadrupole resonance, muon spin resonance, etc.) that exploit the different magnetic or electric character of the atomic nuclei for the impurity and the host in the hybrid system. Finally, the random implantation of the muons in the sample can provide information of the 
relaxation time in different domains with unequal dopant concentration, which may be nonuniform. For the given problem, the differences in the resonant response can give relevant information about the distribution of the local fields and the occurrence of local order and provide access to the dynamical response within doped domains. The use of local spectroscopic resonance methods has been widely demonstrated to be successful when probing the nature and the evolution of the ground state in the presence of spin vacancies both for ordered and disordered magnetic configurations [84-87].

In summary, this study highlights the role of spin defects that lead to orbital dilution in spin-orbital systems. Using an example of $3 d^{3}$ impurities in a $4 d^{4}$ (or $3 d^{2}$ ) host, we have shown that impurities change radically the spin-orbital order around them, independently of the parameter regime. As a general feature, we have found that doped $3 d^{3}$ ions within the host with spin-orbital order have frustrated spins and polarize the orbitals of the host when the impurity-host exchange as well as Hund's exchange at the impurity are both sufficiently large. This remarkable trend is independent of doping and is expected to lead to global changes of spin-orbital order in doped materials. While the latter effect is robust, we argue that the long-range spin fluctuations resulting from the translational invariance of the system will likely prevent the ground state from being macroscopically degenerate, so if the impurity spins in one unit cell happen to choose its polarization, then the others will follow. On the contrary, in the regime of weak Hund's exchange, $3 d^{3}$ ions act as spin defects that not only order antiferromagnetically with respect to their neighbors but also induce doublons in inactive orbitals.

Finally, we remark that this behavior with switching between inactive and active orbitals by an orbitally neutral impurity may lead to multiple interesting phenomena at macroscopic doping when global modifications of the spinorbital order are expected to occur. Most of the results were obtained in the classical approximation, but we have shown that modifications due to spin-orbit coupling do not change the main conclusion. We note that this generic treatment and the general questions addressed here, such as the release of frustration for competing spin structures due to periodic impurities, are relevant to double perovskites [88]. While the local orbital polarization should be similar, it is challenging to investigate disordered impurities, both theoretically and in experiment, to find out whether their influence on the global spin-orbital order in the host is equally strong.

\section{ACKNOWLEDGMENTS}

We thank Maria Daghofer and Krzysztof Wohlfeld for insightful discussions. W. B. and A. M. O. kindly acknowledge support by the Polish National Science Center (NCN) under Project No. 2012/04/A/ST3/00331. W. B. was also supported by the Foundation for Polish Science (FNP) within the START program. M. C. acknowledges funding from the EU-FP7/2007-2013 under Grant Agreement No. 264098-MAMA.

\section{APPENDIX A: DERIVATION OF $3 d-4 d$ SUPEREXCHANGE}

Here, we present the details of the derivation of the lowenergy spin-orbital Hamiltonian for the $3 d^{3}-4 d^{4}$ bonds around the impurity at site $i$. $\mathcal{H}_{3 d-4 d}(i)$ follows from the perturbation theory, as given in Eq. (10). Here, we consider a single $3 d^{3}-4 d^{4}$ bond $\langle i j\rangle$. Two contributions to the effective Hamiltonian follow from charge excitations: (i) $\mathcal{H}_{J, 43}^{(\gamma)}(i, j)$ due to $d_{i}^{3} d_{j}^{4} \leftrightharpoons d_{i}^{4} d_{j}^{3}$ and (ii) $\mathcal{H}_{J, 25}^{(\gamma)}(i, j)$ due to $d_{i}^{3} d_{j}^{4} \leftrightharpoons d_{i}^{2} d_{j}^{5}$. Therefore, the low-energy Hamiltonian is

$$
\mathcal{H}_{J}^{(\gamma)}(i, j)=\mathcal{H}_{J, 43}^{(\gamma)}(i, j)+\mathcal{H}_{J, 25}^{(\gamma)}(i, j)
$$

Consider first the processes that conserve the number of doubly occupied orbitals $d_{i}^{3} d_{j}^{4} \leftrightharpoons d_{i}^{4} d_{j}^{3}$. Then, by means of spin and orbital projectors, it is possible to express $\mathcal{H}_{J, 43}^{(\gamma)}(i, j)$ for $i=1$ and $j=2$ as

$$
\begin{aligned}
\mathcal{H}_{J, 43}^{(\gamma)}(1,2) & =-\left(\vec{S}_{1} \cdot \vec{S}_{2}\right) \frac{t^{2}}{18}\left\{\frac{4}{\Delta}-\frac{7}{\Delta+3 J_{2}^{H}}-\frac{3}{\Delta+5 J_{2}^{H}}\right\} \\
& +D_{2}^{(\gamma)}\left(\vec{S}_{1} \cdot \vec{S}_{2}\right) \frac{t^{2}}{18}\left\{\frac{4}{\Delta}-\frac{1}{\Delta+3 J_{2}^{H}}+\frac{3}{\Delta+5 J_{2}^{H}}\right\} \\
& +\left(D_{2}^{(\gamma)}-1\right) \frac{t^{2}}{12}\left\{\frac{8}{\Delta}+\frac{1}{\Delta+3 J_{2}^{H}}-\frac{3}{\Delta+5 J_{2}^{H}}\right\},
\end{aligned}
$$

with the excitation energy $\Delta$ defined in Eq. (11). The resulting effective $3 d-4 d$ exchange in Eq. (A2) consists of three terms: (i) The first one does not depend on the orbital configuration of the $4 d$ atom, and it can be FM or AF depending on the values $\Delta$ and the Hund's exchange on the $3 d$ ion. In particular, if $\Delta$ is the largest or the smallest energy scale, the coupling will be either AF or FM, respectively. (ii) The second term has an explicit dependence on the occupation of the doublon on the $4 d$ atom via the projecting operator $D_{2}^{(\gamma)}$. This dependence implies that a magnetic exchange is possible only if the doublon occupies the inactive orbital for a bond along a given direction $\gamma$. Unlike in the first term, the sign of this interaction is always positive, favoring an AF configuration at any strength of $\Delta$ and $J_{1}^{H}$. (iii) Finally, the last term describes the effective processes that do not depend on the spin states on the $3 d$ and $4 d$ atoms. This contribution is of a pure orbital nature, as it originates from the hopping between $3 d$ and $4 d$ atoms without affecting their spin configuration, and for this 
reason favors the occupation of active $t_{2 g}$ orbitals along the bond by the doublon.

Within the same scheme, we have derived the effective spin-orbital exchange that originates from the chargetransfer processes of the type $3 d_{1}^{3} 4 d_{2}^{4} \leftrightharpoons 3 d_{i}^{2} 4 d_{j}^{5}$, $\mathcal{H}_{J, 25}^{(\gamma)}(1,2)$. The effective low-energy contribution to the Hamiltonian for $i=1$ and $j=2$ reads

$$
\begin{aligned}
\mathcal{H}_{J, 25}^{(\gamma)}(1,2) & \frac{t^{2}}{U_{1}+U_{2}-\left(\Delta+3 J_{2}^{H}-2 J_{1}^{H}\right)} \\
& \times\left\{\frac{1}{3} D_{2}^{(\gamma)}\left(\vec{S}_{1} \cdot \vec{S}_{2}\right)+\frac{1}{3}\left(\vec{S}_{1} \cdot \vec{S}_{2}\right)-\frac{1}{2}\left(D_{2}^{(\gamma)}+1\right)\right\} .
\end{aligned}
$$

By inspection of the spin structure involved in the elemental processes that generate $\mathcal{H}_{J, 25}^{(\gamma)}(1,2)$, one can note that it is always AF independently of the orbital configuration on the $4 d$ atom exhibiting with a larger spin exchange and an orbital energy gain if the doublon is occupying the inactive orbital along a given bond. We have verified that the amplitude of the exchange terms in $\mathcal{H}_{J, 25}^{(\gamma)}(1,2)$ is much smaller than the ones that enter in $\mathcal{H}_{J, 43}^{(\gamma)}(1,2)$, which justifies that one may simplify Eq. (A1) for $i=1$ and $j=2$ to

$$
\mathcal{H}_{J}^{(\gamma)}(1,2) \simeq \mathcal{H}_{J, 43}^{(\gamma)}(1,2)
$$

and neglect $\mathcal{H}_{J .25}^{(\gamma)}(1,2)$ terms altogether. This approximation is used in Sec. II.

\section{APPENDIX B: ORBITAL OPERATORS IN THE $L$ BASIS}

The starting point to express the orbital operators appearing in the spin-orbital superexchange model (17) is the relation between quenched $|a\rangle_{i},|b\rangle_{i}$, and $|c\rangle_{i}$ orbitals at site $i$ and the eigenvectors $|1\rangle_{i},|0\rangle_{i}$, and $|-1\rangle_{i}$ of the angular momentum operator $L_{i}^{z}$. These relations are known to be

$$
\begin{aligned}
|a\rangle_{i} & =\frac{1}{\sqrt{2}}\left(|1\rangle_{i}+|-1\rangle_{i}\right), \\
|b\rangle_{i} & =\frac{-i}{\sqrt{2}}\left(|1\rangle_{i}-|-1\rangle_{i}\right), \\
|c\rangle_{i} & =|0\rangle_{i} .
\end{aligned}
$$

From these equations, we can immediately get the occupation-number operators for the doublon

$$
\begin{aligned}
& D_{i}^{(a)}=a_{i}^{\dagger} a_{i}=|a\rangle_{i}\left\langle\left. a\right|_{i}=1-\left(L_{i}^{x}\right)^{2},\right. \\
& D_{i}^{(b)}=b_{i}^{\dagger} b_{i}=|b\rangle_{i}\left\langle\left. b\right|_{i}=1-\left(L_{i}^{y}\right)^{2},\right. \\
& D_{i}^{(c)}=c_{i}^{\dagger} c_{i}=|c\rangle_{i}\left\langle\left. c\right|_{i}=1-\left(L_{i}^{z}\right)^{2}\right.
\end{aligned}
$$

and the related $\left\{n_{i}^{(\gamma)}\right\}$ operators

$$
\begin{aligned}
& n_{i}^{(a)}=b_{i}^{\dagger} b_{i}+c_{i}^{\dagger} c_{i}=\left(L_{i}^{x}\right)^{2}, \\
& n_{i}^{(b)}=c_{i}^{\dagger} c_{i}+a_{i}^{\dagger} a_{i}=\left(L_{i}^{y}\right)^{2}, \\
& n_{i}^{(c)}=a_{i}^{\dagger} a_{i}+b_{i}^{\dagger} b_{i}=\left(L_{i}^{z}\right)^{2} .
\end{aligned}
$$

The doublon hopping operators have a slightly different structure that reflects their noncommutativity, i.e.,

$$
\begin{aligned}
a_{i}^{\dagger} b_{i} & =|a\rangle_{i}\left\langle\left. b\right|_{i}=i L_{i}^{y} L_{i}^{x},\right. \\
b_{i}^{\dagger} c_{i} & =|b\rangle_{i}\left\langle\left. c\right|_{i}=i L_{i}^{z} L_{i}^{y},\right. \\
c_{i}^{\dagger} a_{i} & =|c\rangle_{i}\left\langle\left. a\right|_{i}=i L_{i}^{x} L_{i}^{z} .\right.
\end{aligned}
$$

These relations are sufficient to write the superexchange Hamiltonian for the host-host and impurity-host bonds in the $\left\{L_{i}^{x}, L_{i}^{y}, L_{i}^{z}\right\}$ operator basis for the orbital part. However, in practice, it is more convenient to work with real operators $\left\{L_{i}^{+}, L_{i}^{-}, L_{i}^{z}\right\}$ rather than with the original ones $\left\{L_{i}^{x}, L_{i}^{y}, L_{i}^{z}\right\}$. Thus, we write the final relations, which we use for the numerical calculations in terms of these operators

$$
\begin{aligned}
D_{i}^{(a)} & =-\frac{1}{4}\left[\left(L_{i}^{+}\right)^{2}+\left(L_{i}^{-}\right)^{2}\right]+\frac{1}{2}\left(L_{i}^{z}\right)^{2}, \\
D_{i}^{(b)} & =\frac{1}{4}\left[\left(L_{i}^{+}\right)^{2}+\left(L_{i}^{-}\right)^{2}\right]+\frac{1}{2}\left(L_{i}^{z}\right)^{2}, \\
D_{i}^{(c)} & =1-\left(L_{i}^{z}\right)^{2} ;
\end{aligned}
$$

for the doublon occupation numbers and going directly to the orbital $\vec{\tau}_{i}$ operators, we find that

$$
\begin{aligned}
\tau_{i}^{+(a)} & =\frac{1}{2}\left(L_{i}^{-}-L_{i}^{+}\right) L_{i}^{z}, \\
\tau_{i}^{+(b)} & =\frac{-i}{2} L_{i}^{z}\left(L_{i}^{+}+L_{i}^{-}\right), \\
\tau_{i}^{+(c)} & =\frac{i}{4}\left[\left(L_{i}^{+}\right)^{2}-\left(L_{i}^{-}\right)^{2}\right]-\frac{i}{2} L_{i}^{z}
\end{aligned}
$$

for the off-diagonal part and

$$
\begin{aligned}
\tau_{i}^{z(a)} & =\frac{1}{8}\left[\left(L_{i}^{+}\right)^{2}+\left(L_{i}^{-}\right)^{2}\right]+\frac{3}{4}\left(L_{i}^{z}\right)^{2}-\frac{1}{2}, \\
\tau_{i}^{z(b)} & =\frac{1}{8}\left[\left(L_{i}^{+}\right)^{2}+\left(L_{i}^{-}\right)^{2}\right]-\frac{3}{4}\left(L_{i}^{z}\right)^{2}+\frac{1}{2}, \\
\tau_{i}^{z(c)} & =-\frac{1}{4}\left[\left(L_{i}^{+}\right)^{2}+\left(L_{i}^{-}\right)^{2}\right]
\end{aligned}
$$


for the diagonal one. Note that the complex phase in $\tau_{i}^{+(b)}$ and $\tau_{i}^{+(c)}$ is irrelevant and can be omitted here, as $\tau_{i}^{+(\gamma)}$ is always accompanied by $\tau_{j}^{-(\gamma)}$ on a neighboring site, being a consequence of the cubic symmetry in the orbital part of the superexchange Hamiltonian, and it can be altered by the presence of a distortion, e.g., octahedral rotation. For completeness, we also give the backward relation between angular momentum components $\left\{L_{i}^{\alpha}\right\}$ with $\alpha=x, y, z$ and the orbital operators $\left\{\tau_{i}^{\alpha(\gamma)}\right\}$; which are the following:

$$
\begin{aligned}
& L_{i}^{x}=2 \tau_{i}^{x(a)}, \\
& L_{i}^{y}=2 \tau_{i}^{x(b)}, \\
& L_{i}^{z}=2 \tau_{i}^{y(c)} .
\end{aligned}
$$

[1] M. Imada, A. Fujimori, and Y. Tokura, Metal-Insulator Transitions, Rev. Mod. Phys. 70, 1039 (1998).

[2] E. Berg, E. Fradkin, S. A. Kivelson, and J. M. Tranquada, Striped Superconductors: How Spin, Charge and Superconducting Orders Intertwine in the Cuprates, New J. Phys. 11, 115004 (2011).

[3] P. A. Lee, N. Nagaosa, and X.-G. Wen, Doping a Mott Insulator: Physics of High-Temperature Superconductivity, Rev. Mod. Phys. 78, 17 (2006).

[4] M. Vojta, Lattice Symmetry Breaking in Cuprate Superconductors: Stripes, Nematics, and Superconductivity, Adv. Phys. 58, 699 (2009).

[5] K. Yamada, C. H. Lee, K. Kurahashi, J. Wada, S. Wakimoto, S. Ueki, H. Kimura, Y. Endoh, S. Hosoya, G. Shirane, R. J. Birgeneau, M. Greven, M. A. Kastner, and Y. J. Kim, Doping Dependence of the Spatially Modulated Dynamical Spin Correlations and the Superconducting-Transition Temperature in $\mathrm{La}_{2-x} \mathrm{Sr}_{x} \mathrm{CuO}_{4}$, Phys. Rev. B 57, 6165 (1998).

[6] M. Fleck, A. I. Lichtenstein, and A. M. Oleś, Spectral Properties and Pseudogap in the Stripe Phases of Cuprate Superconductors, Phys. Rev. B 64, 134528 (2001).

[7] P. Wróbel and A. M. Oleś, Ferro-orbitally Ordered Stripes in Systems with Alternating Orbital Order, Phys. Rev. Lett. 104, 206401 (2010).

[8] Q. Li, K. E. Gray, S. B. Wilkins, M. G. Fernandez, S. Rosenkranz, H. Zheng, and J. F. Mitchell, Prediction and Experimental Evidence for Thermodynamically Stable Charged Orbital Domain Walls, Phys. Rev. X 4, 031028 (2014).

[9] J. Zaanen and A. M. Oleś, Carriers Binding to Excitons: Crystal-Field Excitations in Doped Mott-Hubbard Insulators, Phys. Rev. B 48, 7197 (1993).

[10] Y. Tokura and N. Nagaosa, Orbital Physics in TransitionMetal Oxides, Science 288, 462 (2000).

[11] K. I. Kugel and D. I. Khomskii, The Jahn-Teller Effect and Magnetism: Transition Metal Compounds, Sov. Phys. Usp. 25, 231 (1982).
[12] A. M. Oleś, G. Khaliullin, P. Horsch, and L. F. Feiner, Fingerprints of Spin-Orbital Physics in Cubic Mott Insulators: Magnetic Exchange Interactions and Optical Spectral Weights, Phys. Rev. B 72, 214431 (2005).

[13] K. Wohlfeld, M. Daghofer, S. Nishimoto, G. Khaliullin, and J. van den Brink, Intrinsic Coupling of Orbital Excitations to Spin Fluctuations in Mott Insulators, Phys. Rev. Lett. 107, 147201 (2011).

[14] L. F. Feiner, A. M. Oleś, and J. Zaanen, Quantum Melting of Magnetic Order due to Orbital Fluctuations, Phys. Rev. Lett. 78, 2799 (1997); Quantum Disorder versus Orderout-of-Disorder in the Kugel-Khomskii Model, J. Phys. Condens. Matter 10, L555 (1998).

[15] G. Khaliullin and S. Maekawa, Orbital Liquid in ThreeDimensional Mott Insulator: $\mathrm{LaTiO}_{3}$, Phys. Rev. Lett. 85, 3950 (2000).

[16] G. Khaliullin, Orbital Order and Fluctuations in Mott Insulators, Prog. Theor. Phys. Suppl. 160, 155 (2005).

[17] L. Balents, Spin Liquids in Frustrated Magnets, Nature (London) 464, 199 (2010).

[18] L. F. Feiner and A. M. Oleś, Orbital Liquid in Ferromagnetic Manganites: The Orbital Hubbard Model for $e_{g}$ Electrons, Phys. Rev. B 71, 144422 (2005).

[19] S. Nakatsuji, K. Kuga, K. Kimura, R. Satake, N. Katayama, E. Nishibori, H. Sawa, R. Ishii, M. Hagiwara, F. Bridges, T. U. Ito, W. Higemoto, Y. Karaki, M. Halim, A. A. Nugroho, J. A. Rodriguez-Rivera, M. A. Green, and C. Broholm, Spin-Orbital Short-Range Order on a Honeycomb-Based Lattice, Science 336, 559 (2012).

[20] J. A. Quilliam, F. Bert, E. Kermarrec, C. Payen, C. GuillotDeudon, P. Bonville, C. Baines, H. Luetkens, and P. Mendels, Singlet Ground State of the Quantum Antiferromagnet $\mathrm{Ba}_{3} \mathrm{CuSb}_{2} \mathrm{O}_{9}$, Phys. Rev. Lett. 109, 117203 (2012).

[21] B. Normand and A. M. Oleś, Frustration and Entanglement in the $t_{2 g}$ Spin-Orbital Model on a Triangular Lattice: Valence-Bond and Generalized Liquid States, Phys. Rev. B 78, 094427 (2008).

[22] P. Corboz, M. Lajkó, A. M. Laüchli, K. Penc, and F. Mila, Spin-Orbital Quantum Liquid on the Honeycomb Lattice, Phys. Rev. X 2, 041013 (2012).

[23] J. Nasu and S. Ishihara, Dynamical Jahn-Teller Effect in a Spin-Orbital Coupled System, Phys. Rev. B 88, 094408 (2013).

[24] A. Smerald and F. Mila, Exploring the Spin-Orbital Ground State of $\mathrm{Ba}_{3} \mathrm{CuSb}_{2} \mathrm{O}_{9}$, Phys. Rev. B 90, 094422 (2014).

[25] V. Fritsch, J. Hemberger, N. Büttgen, E.-W. Scheidt, H.-A. Krug von Nidda, A. Loidl, and V. Tsurkan, Spin and Orbital Frustration in $\mathrm{MnSc}_{2} \mathrm{~S}_{2}$ and $\mathrm{FeSc}_{2} \mathrm{~S}_{4}$, Phys. Rev. Lett. 92 , 116401 (2004).

[26] G. Chen, L. Balents, and A. P. Schnyder, Spin-Orbital Singlet and Quantum Critical Point on the Diamond Lattice: $\mathrm{FeSc}_{2} \mathrm{~S}_{4}$, Phys. Rev. Lett. 102, 096406 (2009).

[27] L. Mittelstädt, M. Schmidt, Z. Wang, F. Mayr, V. Tsurkan, P. Lunkenheimer, D. Ish, L. Balents, J. Deisenhofer, and A. Loidl, Spin-Orbiton and Quantum Criticality in $\mathrm{FeSc}_{2} \mathrm{~S}_{4}$, arXiv:1410.6459 [Phys. Rev. B (to be published)].

[28] F. Vernay, K. Penc, P. Fazekas, and F. Mila, Orbital Degeneracy as a Source of Frustration in $\mathrm{LiNiO}_{2}$, Phys. Rev. B 70, 014428 (2004). 
[29] W. Brzezicki, J. Dziarmaga, and A. M. Oleś, Noncollinear Magnetic Order Stabilized by Entangled Spin-Orbital Fluctuations, Phys. Rev. Lett. 109, 237201 (2012).

[30] W. Brzezicki, J. Dziarmaga, and A. M. Oleś, Exotic Spin Orders Driven by Orbital Fluctuations in the KugelKhomskii Model, Phys. Rev. B 87, 064407 (2013).

[31] P. Czarnik and J. Dziarmaga, Striped Critical Spin Liquid in a Spin-Orbital Entangled RVB State in a Projected Entangled-Pair State Representation, Phys. Rev. B 91, 045101 (2015).

[32] F. Reynaud, D. Mertz, F. Celestini, J. M. Debierre, A. M. Ghorayeb, P. Simon, A. Stepanov, J. Voiron, and C. Delmas, Orbital Frustration at the Origin of the Magnetic Behavior in $\mathrm{LiNiO}_{2}$, Phys. Rev. Lett. 86, 3638 (2001).

[33] A. Reitsma, L. F. Feiner, and A. M. Oleś, Orbital and Spin Physics in $\mathrm{LiNiO}_{2}$ and $\mathrm{NaNiO}_{2}$, New J. Phys. 7, 121 (2005).

[34] A. M. Oleś, Fingerprints of Spin-Orbital Entanglement in Transition Metal Oxides, J. Phys. Condens. Matter 24, 313201 (2012).

[35] E. Dagotto, T. Hotta, and A. Moreo, Colossal Magnetoresistant Materials: The Key Role of Phase Separation, Phys. Rep. 344, 1 (2001).

[36] E. Dagotto, Open Questions in CMR Manganites, Relevance of Clustered States and Analogies with Other Compounds Including the Cuprates, New J. Phys. 7, 67 (2005).

[37] Y. Tokura, Critical Features of Colossal Magnetoresistive Manganites, Rep. Prog. Phys. 69, 797 (2006).

[38] L. F. Feiner and A. M. Oleś, Electronic Origin of Magnetic and Orbital Ordering in Insulating $\mathrm{LaMnO}_{3}$, Phys. Rev. B 59, 3295 (1999).

[39] R. Kilian and G. Khaliullin, Orbital Polarons in the MetalInsulator Transition of Manganites, Phys. Rev. B 60, 13458 (1999).

[40] M. Cuoco, C. Noce, and A. M. Oleś, Origin of the Optical Gap in Half-Doped Manganites, Phys. Rev. B 66, 094427 (2002).

[41] A. Weisse and H. Fehske, Microscopic Modelling of Doped Manganites, New J. Phys. 6, 158 (2004).

[42] M. Daghofer, A. M. Oleś, and W. von der Linden, Orbital Polarons versus Itinerant $e_{g}$ Electrons in Doped Manganites, Phys. Rev. B 70, 184430 (2004).

[43] J. Geck, P. Wochner, S. Kiele, R. Klingeler, P. Reutler, A. Revcolevschi, and B. Büchner, Orbital Polaron Lattice Formation in Lightly Doped $\mathrm{La}_{1-x} \mathrm{Sr}_{x} \mathrm{MnO}_{3}$, Phys. Rev. Lett. 95, 236401 (2005).

[44] A. M. Oleś and G. Khaliullin, Dimensional Crossover and the Magnetic Transition in Electron Doped Manganites, Phys. Rev. B 84, 214414 (2011).

[45] Y. M. Sheu, S. A. Trugman, L. Yan, J. Qi, Q. X. Jia, A. J. Taylor, and R. P. Prasankumar, Polaronic Transport Induced by Competing Interfacial Magnetic Order in a $\mathrm{La}_{0.7} \mathrm{Ca}_{0.3} \mathrm{MnO}_{3} / \mathrm{BiFeO}_{3}$ Heterostructure, Phys. Rev. X 4, 021001 (2014).

[46] P.-G. de Gennes, Effects of Double Exchange in Magnetic Crystals, Phys. Rev. 118, 141 (1960).

[47] G. Khaliullin and R. Kilian, Theory of Anomalous Magnon Softening in Ferromagnetic Manganites, Phys. Rev. B 61, 3494 (2000).
[48] A. M. Oleś and L.F. Feiner, Why Spin Excitations in Metallic Ferromagnetic Manganites Are Isotropic, Phys. Rev. B 65, 052414 (2002).

[49] H. Sakai, S. Ishiwata, D. Okuyama, A. Nakao, H. Nakao, Y. Murakami, Y. Taguchi, and Y. Tokura, Electron Doping in the Cubic Perovskite $\mathrm{SrMnO}_{3}$ : Isotropic Metal versus Chainlike Ordering of Jahn-Teller Polarons, Phys. Rev. B 82, 180409 (2010).

[50] G. Khaliullin, P. Horsch, and A. M. Oleś, Spin Order due to Orbital Fluctuations: Cubic Vanadates, Phys. Rev. Lett. 86, 3879 (2001).

[51] G. Khaliullin, P. Horsch, and A. M. Oleś, Theory of Optical Spectral Weights in Mott Insulators with Orbital Degrees of Freedom, Phys. Rev. B 70, 195103 (2004).

[52] P. Horsch, A. M. Oleś, L. F. Feiner, and G. Khaliullin, Evolution of Spin-Orbital-Lattice Coupling in the $\mathrm{RVO}_{3}$ Perovskites, Phys. Rev. Lett. 100, 167205 (2008).

[53] C. Ulrich, G. Khaliullin, J. Sirker, M. Reehuis, M. Ohl, S. Miyasaka, Y. Tokura, and B. Keimer, Magnetic Neutron Scattering Study of $\mathrm{YVO}_{3}$ : Evidence for an Orbital Peierls State, Phys. Rev. Lett. 91, 257202 (2003).

[54] J.-S. Zhou, J. B. Goodenough, J.-Q. Yan, and Y. Ren, Superexchange Interaction in Orbitally Fluctuating $\mathrm{RVO}_{3}$, Phys. Rev. Lett. 99, 156401 (2007).

[55] M. Reehuis, C. Ulrich, K. Prokeš, S. Mat'aš, J. Fujioka, S. Miyasaka, Y. Tokura, and B. Keimer, Structural and Magnetic Phase Transitions of the Orthovanadates $\mathrm{RVO}_{3}$ $(R=\mathrm{Dy}, \mathrm{Ho}, \mathrm{Er})$ as seen via Neutron Diffraction, Phys. Rev. B 83, 064404 (2011).

[56] J. Fujioka, T. Yasue, S. Miyasaka, Y. Yamasaki, T. Arima, H. Sagayama, T. Inami, K. Ishii, and Y. Tokura, Critical Competition between two Distinct Orbital-Spin Ordered States in Perovskite Vanadates, Phys. Rev. B 82, 144425 (2010).

[57] P. Horsch, G. Khaliullin, and A. M. Oleś, Dimerization versus Orbital-Moment Ordering in a Mott Insulator $\mathrm{YVO}_{3}$, Phys. Rev. Lett. 91, 257203 (2003).

[58] J. Fujioka, S. Miyasaka, and Y. Tokura, Doping Variation of Anisotropic Charge and Orbital Dynamics in $\mathrm{Y}_{1-x} \mathrm{Ca}_{x} \mathrm{VO}_{3}$ : Comparison with $\mathrm{La}_{1-x} \mathrm{Sr}_{x} \mathrm{VO}_{3}$, Phys. Rev. B 77, 144402 (2008).

[59] P. Horsch and A. M. Oleś, Defect States and Spin-Orbital Physics in Doped Vanadates $\mathrm{Y}_{1-x} \mathrm{Ca}_{x} \mathrm{VO}_{3}$, Phys. Rev. B 84, 064429 (2011).

[60] A. Avella, P. Horsch, and A. M. Oleś, Defect States and Excitations in a Mott Insulator with Orbital Degrees of Freedom: Mott-Hubbard Gap versus Optical and Transport Gaps in Doped Systems, Phys. Rev. B 87, 045132 (2013).

[61] T. Hotta, Orbital Ordering Phenomena in $d$ - and $f$-Electron Systems, Rep. Prog. Phys. 69, 2061 (2006).

[62] T. Mizokawa, L. H. Tjeng, G. A. Sawatzky, G. Ghiringhelli, O. Tjernberg, N. B. Brookes, H. Fukazawa, S. Nakatsuji, and Y. Maeno, Spin-Orbit Coupling in the Mott Insulator $\mathrm{Ca}_{2} \mathrm{RuO}_{4}$, Phys. Rev. Lett. 87, 077202 (2001).

[63] J. S. Lee, Y. S. Lee, T. W. Noh, S.-J. Oh, J. Yu, S. Nakatsuji, H. Fukazawa, and Y. Maeno, Electron and Orbital Correlations in $\mathrm{Ca}_{2-x} \mathrm{Sr}_{x} \mathrm{RuO}_{4}$ Probed by Optical Spectroscopy, Phys. Rev. Lett. 89, 257402 (2002). 
[64] A. Koga, N. Kawakami, T. M. Rice, and M. Sigrist, OrbitalSelective Mott Transitions in the Degenerate Hubbard Model, Phys. Rev. Lett. 92, 216402 (2004).

[65] Z. Fang, K. Terakura, and N. Nagaosa, Orbital Physics in Ruthenates: First-Principles Study, New J. Phys. 7, 66 (2005).

[66] T. Sugimoto, D. Ootsuki, and T. Mizokawa, Impact of Local Lattice Disorder on Spin and Orbital Orders in $\mathrm{Ca}_{2-x} \mathrm{Sr}_{x} \mathrm{RuO}_{4}$, Phys. Rev. B 84, 064429 (2011).

[67] M. Cuoco, F. Forte, and C. Noce, Probing Spin-OrbitalLattice Correlations in $4 d^{4}$ Systems, Phys. Rev. B 73, 094428 (2006).

[68] G. Khaliullin, Excitonic Magnetism in Van Vleck-type $d^{4}$ Mott Insulators, Phys. Rev. Lett. 111, 197201 (2013); A. Akbari and G. Khaliullin, Magnetic Excitations in a Spin-Orbit-Coupled $d^{4}$ Mott Insulator on the Square Lattice, Phys. Rev. B 90, 035137 (2014).

[69] C. N. Veenstra, Z.-H. Zhu, M. Raichle, B. M. Ludbrook, A. Nicolaou, B. Slomski, G. Landolt, S. Kittaka, Y. Maeno, J. H. Dil, I. S. Elfimov, M. W. Haverkort, and A. Damascelli, Spin-Orbital Entanglement and the Breakdown of Singlets and Triplets in $\mathrm{Sr}_{2} \mathrm{RuO}_{4}$ Revealed by Spin- and AngleResolved Photoemission Spectroscopy, Phys. Rev. Lett. 112, 127002 (2014).

[70] T. Tanaka and S. Ishihara, Dilution Effects in TwoDimensional Quantum Orbital Systems, Phys. Rev. Lett. 98, 256402 (2007).

[71] T. F. Qi, O. B. Korneta, S. Parkin, L. E. De Long, P. Schlottmann, and G. Cao, Negative Volume Thermal Expansion via Orbital and Magnetic Orders in $\mathrm{Ca}_{2} \mathrm{Ru}_{1-x} \mathrm{Cr}_{x} \mathrm{O}_{4} \quad(0<x<0.13)$, Phys. Rev. Lett. 105, 177203 (2010).

[72] G. Cao, S. Chikara, X. N. Lin, E. Elhami, V. Durairaj, and P. Schlottmann, Itinerant Ferromagnetism to Insulating Antiferromagnetism: A Magnetic and Transport Study of Single Crystal $\mathrm{SrRu}_{1-x} \mathrm{Mn}_{x} \mathrm{O}_{3}(0 \leq x<0.60)$, Phys. Rev. B 71, 035104 (2005).

[73] M. A. Hossain, B. Bohnenbuck, Y. D. Chuang, M. W. Haverkort, I. S. Elfimov, A. Tanaka, A. G. Cruz Gonzalez, Z. Hu, H.-J. Lin, C. T. Chen, R. Mathieu, Y. Tokura, Y. Yoshida, L. H. Tjeng, Z. Hussain, B. Keimer, G. A. Sawatzky, and A. Damascelli, Mott versus Slater-Type Metal-Insulator Transition in Mn-Substituted $\mathrm{Sr}_{3} \mathrm{Ru}_{2} \mathrm{O}_{7}$, Phys. Rev. B 86, 041102(R) (2012).

[74] M. A. Hossain, Z. Hu, M. W. Haverkort, T. Burnus, C. F. Chang, S. Klein, J. D. Denlinger, H.-J. Lin, C. T. Chen, R. Mathieu, Y. Kaneko, Y. Tokura, S. Satow, Y. Yoshida, H. Takagi, A. Tanaka, I. S. Elfimov, G. A. Sawatzky, L. H. Tjeng, and A. Damascelli, Crystal-Field Level Inversion in Lightly Mn-Doped $\mathrm{Sr}_{3} \mathrm{Ru}_{2} \mathrm{O}_{7}$, Phys. Rev. Lett. 101, 016404 (2008).

[75] D. Mesa, F. Ye, S. Chi, J. A. Fernandez-Baca, W. Tian, B. Hu, R. Jin, E. W. Plummer, and J. Zhang, Single-Bilayer E-Type Antiferromagnetism in Mn-Substituted $\mathrm{Sr}_{3} \mathrm{Ru}_{2} \mathrm{O}_{7}$ :
Neutron Scattering Study, Phys. Rev. B 85, 180410(R) (2012).

[76] S. A. J. Kimber, J. A. Rodgers, H. Wu, C. A. Murray, D. N. Argyriou, A. N. Fitch, D. I. Khomskii, and J. P. Attfield, Metal-Insulator Transition and Orbital Order in $\mathrm{PbRuO}_{3}$, Phys. Rev. Lett. 102, 046409 (2009).

[77] J. F. Annett, G. Litak, B. L. Györffy, and K. I. Wysokiński, Spin-Orbit Coupling and Symmetry of the Order Parameter in Strontium Ruthenate, Phys. Rev. B 73, 134501 (2006).

[78] A. M. Oleś, Antiferromagnetism and Correlation of Electrons in Transition Metals, Phys. Rev. B 28, 327 (1983).

[79] A. B. Harris, T. Yildirim, A. Aharony, O. Entin-Wohlman, and I. Ya. Korenblit, Unusual Symmetries in the KugelKhomskii Hamiltonian, Phys. Rev. Lett. 91, 087206 (2003).

[80] M. Daghofer, K. Wohlfeld, A. M. Oleś, E. Arrigoni, and P. Horsch, Absence of Hole Confinement in TransitionMetal Oxides with Orbital Degeneracy, Phys. Rev. Lett. 100, 066403 (2008).

[81] M. Cuoco, F. Forte, and C. Noce, Interplay of Coulomb Interactions and c-Axis Octahedra Distortions in SingleLayer Ruthenates, Phys. Rev. B 74, 195124 (2006).

[82] P. Marra, K. Wohlfeld, and J. van den Brink, Unraveling Orbital Correlations with Magnetic Resonant Inelastic X-Ray Scattering, Phys. Rev. Lett. 109, 117401 (2012).

[83] M. A. Hossain, I. Zegkinoglou, Y.-D. Chuang, J. Geck, B. Bohnenbuck, A. G. Cruz Gonzalez, H.-H. Wu, C. SchüßlerLangeheine, D. G. Hawthorn, J. D. Denlinger, R. Mathieu, Y. Tokura, S. Satow, H. Takagi, Y. Yoshida, Z. Hussain, B. Keimer, G. A. Sawatzky, and A. Damascelli, Electronic Superlattice Revealed by Resonant Scattering from Random Impurities in $\mathrm{Sr}_{3} \mathrm{Ru}_{2} \mathrm{O}_{7}$, Sci. Rep. 3, 2299 (2013).

[84] L. Limot, P. Mendels, G. Collin, C. Mondelli, B. Ouladdiaf, H. Mutka, N. Blanchard, and M. Mekata, Susceptibility and Dilution Effects of the Kagomé Bilayer Geometrically Frustrated Network: A Ga NMR Study of $\mathrm{SrCr}_{9 p} \mathrm{Ga}_{12-9 p} \mathrm{O}_{19}$, Phys. Rev. B 65, 144447 (2002).

[85] J. Bobroff, N. Laflorencie, L. K. Alexander, A. V. Mahajan, B. Koteswararao, and P. Mendels, Impurity-Induced Magnetic Order in Low-Dimensional Spin-Gapped Materials, Phys. Rev. Lett. 103, 047201 (2009).

[86] A. Sen, K. Damle, and R. Moessner, Fractional Spin Textures in the Frustrated Magnet $\mathrm{SrCr}_{9 p} \mathrm{Ga}_{12-9 p} \mathrm{O}_{19}$, Phys. Rev. Lett. 106, 127203 (2011).

[87] P. Bonfà, P. Carretta, S. Sanna, G. Lamura, G. Prando, A. Martinelli, A. Palenzona, M. Tropeano, M. Putti, and R. De Renzi, Magnetic Properties of Spin-Diluted Iron Pnictides from $\mu \mathrm{SR}$ and $N M R$ in $\mathrm{LaFe}_{1-x} \mathrm{Ru}_{x} \mathrm{AsO}$, Phys. Rev. B 85, 054518 (2012).

[88] A. K. Paul, M. Reehuis, V. Ksenofontov, B. Yan, A. Hoser, D. M. Többens, P. M. Abdala, P. Adler, M. Jansen, and C. Felser, Lattice Instability and Competing Spin Structures in the Double Perovskite Insulator $\mathrm{Sr}_{2} \mathrm{FeOsO}_{6}$, Phys. Rev. Lett. 111, 167205 (2013). 